\title{
ON THE DISTRIBUTION OF MULTIPLES OF REAL NUMBERS
}

\author{
TANGUY RIVOAL
}

\section{INTRODUCTION}

We note the integer part of a real number $\alpha$ by $\lfloor\alpha\rfloor$, its fractional part by $\{\alpha\}$ and the nearest integer to $\alpha$ by $\lfloor\alpha\rceil$, with the convention that it is $\lfloor\alpha\rfloor$ if $\alpha \in \mathbb{Z}+\frac{1}{2}$. The distance of $\alpha$ to $\mathbb{Z}$ is $\|\alpha\|:=|\alpha-\lfloor\alpha\rceil|$, whose value does not depend of the above convention.

The two main objects studied in this paper are the following sequences of continuous functions of $\alpha$ :

$$
F_{n}(\alpha):=\sum_{k=1}^{n}\left|k \alpha-\frac{\lfloor k n \alpha\rceil}{n}\right|=\frac{1}{n} \sum_{k=1}^{n}\|n k \alpha\|,
$$

whose value is approximately $\frac{1}{4}$ (and understanding the word "approximately" is the aim of the present work), and its weighted average

$$
G_{s, N}(\alpha):=\sum_{n=1}^{N} \frac{1}{n^{s}} F_{n}(\alpha) .
$$

A priori, $\alpha$ and $s$ can be any real numbers but restriction will be made later.

In a certain sense, $F_{n}(\alpha)$ and $G_{s, N}(\alpha)$ are tools to measure how far the (multi)set $\{\{\alpha\},\{2 \alpha\}, \ldots,\{n \alpha\}\}$ is from being equal to a subset of $\left\{\frac{0}{n}, \frac{1}{n}, \ldots, \frac{n-1}{n}\right\}$. This is a problem related to uniform distribution of the sequence $(\{n \alpha\})_{n}$ and rational approximations of $\alpha$. Before going into the core of the paper in Section 1.2, we set a few definitions and recall some basic facts.

1.1. Lagrange constants and other diophantine statistics. We will occasionaly use the notion of Lagrange constant $L(\alpha)$ of an irrational number $\alpha$. It is defined $\left(^{1}\right)$ as $L(\alpha):=\lim \sup _{q \rightarrow+\infty} \frac{1}{q\|q \alpha\|}$. The smallest value of $L(\alpha)$ is $\sqrt{5}$ and is achieved at $\frac{\sqrt{5}-1}{2}$ and numbers equivalent to it (in the sense of continued fractions, see below). The next smallest value $\sqrt{8}$ is achieved at $\sqrt{2}$ and numbers equivalent to it, etc. Furthermore, the lim sup defining $L(\alpha)$ is achieved along the subsequence $\left(q_{n}\right)_{n}$ of the denominators of the continued fraction of $\alpha$. The set of values of $L(\alpha)$ is the Lagrange spectrum, which was studied a lot in the literature. See [7] for a survey of its properties. That the minimal value of $L$ is

Date: January 2, 2010.

2000 Mathematics Subject Classification. Primary 11K60; Secondary 11J06, 11K70, 42A16.

Key words and phrases. Diophantine approximation, Lagrange spectrum, Continued fractions, Fourier series.

${ }^{1}$ In the literature, Lagrange constants are also defined as $\widehat{L}(\alpha):=\liminf _{q \rightarrow+\infty} q\|q \alpha\|$. This does not change much to the theory because $\widehat{L}(\alpha)=1 / L(\alpha)$. 
achieved at $\frac{\sqrt{5}+1}{2}$ supports the claim that this number (and its equivalents) is the "most irrational number".

Moreover, $L(\alpha)=+\infty$ if and only if, in the continued fraction $\left[a_{0}, a_{1}, a_{2}, \ldots\right]$ of $\alpha$, the sequence of the partial quotients $\left(a_{n}\right)_{n}$ is unbounded; this is due to the inequality $\left|\alpha-\frac{p_{n}}{q_{n}}\right| \leq$ $\frac{1}{a_{n+1} q_{n}^{2}}$. In particular, $L(\alpha)$ is finite for $\alpha$ in a set of measure 0 . The irrationality exponent $m(\alpha)$, another classical diophantine statistic defined in Section 1.3, is equal to 2 almost surely and therefore does not really distinguish irrational numbers. Discrepancy theory leads to two other interesting statistics. Starting from the star-discrepancy $D_{N}^{*}(\alpha):=$ $\sup _{0 \leq x<1}\left(\frac{1}{N} \#\{1 \leq n \leq N:\{n \alpha\} \in[0, x)\}-x\right)$ of the sequence of fractional part $(\{n \alpha\})_{n}$, the quantity

$$
S^{*}(\alpha):=\limsup _{N \rightarrow+\infty} \frac{N}{\log (N)} D_{N}^{*}(\alpha)
$$

has been shown to fill the ray $\left[S^{*}(\sqrt{2}), \infty\right]$ by Baxa [2]. A similar result is not yet known for the discrepancy $D_{N}(\alpha):=\sup _{0 \leq x<y<1}\left(\frac{1}{N} \#\{1 \leq n \leq N:\{n \alpha\} \in[x, y)\}-(y-x)\right)$, although it is expected that the corresponding quantity $S(\alpha)$ fills the ray $\left[S\left(\frac{\sqrt{5}+1}{2}\right), \infty\right]$. However, $S^{*}(\alpha)$ and $S(\alpha)$ are finite if and only if $\sum_{j=1}^{n} a_{j}=\mathcal{O}(n)$, which corresponds to a set of measure 0 because $\sum_{j=1}^{n} a_{j} \gg n \log (n)$ almost surely. In the context of irregularities of distribution of $(\{n \alpha\})_{n}$, we can also mention the "dispersion constant" of Niederreiter [18] and the similar one of Jager-De Jong [12], both of which are minimal at $\frac{\sqrt{5}-1}{2}$ (and its equivalents) and finite for a given irrational number $\alpha$ if and only if $\left(a_{n}\right)_{n}$ is bounded.

All the above mentioned functions present the particularity to be equal to a certain constant almost everywhere. We will show that $G_{s, N}(\alpha)$ enables to distinguish in a subtile way more irrational numbers (where the meaning of "more" depends on $s$ ) and that extremality properties around $\frac{\sqrt{5}-1}{2}$ seem to be also present.

1.2. Description of $F_{n}(\alpha)$ and $G_{s, N}(\alpha)$. The behavior of $F_{n}(\alpha)$ strongly depends on the (ir)rationality of $\alpha$ and also on whether or not $n$ is a denominator of a convergent of $\alpha$. The function $G_{s, N}(\alpha)$ smoothens the dependence on $n$. In this respect, since $0 \leq F_{n}(\alpha) \leq \frac{1}{2}$, it is clear that the sequence $\left(G_{s, N}(\alpha)\right)_{N \geq 1}$ converges as $N \rightarrow+\infty$ for any real number $\alpha$ and any $s>1$. We will no longer consider this case because it gives too much weight to the first values of $\left(F_{n}(\alpha)\right)_{n \geq 1}$ whereas we seek average results.

We will first study the sequence $\left(F_{n}(\alpha)\right)_{n \geq 1}$ whose behavior is not easy to understand, despite what is shown by Figure 1. We will show in Theorem 4 in Section 2 that, in particular, $\left(F_{n}(\alpha)\right)_{n>1}$ tends to be periodic when $\alpha$ is rational. When $\alpha$ is irrational, we will obtain lower and upper bounds for the liminf and limsup of $F_{n}(\alpha)$, in particular $\liminf _{n} F_{n}(\alpha) \leq(2 L(\alpha))^{-1}$. We also observe that each function $F_{n}(\alpha)$ is 1-periodic in $\alpha$ and satisfies the equation $F_{n}(1-\alpha)=F_{n}(\alpha)$; these two properties are also inherited by $G_{s, N}(\alpha)$ and the limiting cases studied in the paper, the first one justifying that we limit ourselves to the case $\alpha \in[0,1]$.

We will investigate in much more details the behavior of the sequence $\left(G_{s, N}(\alpha)\right)_{N>1}$. We will focus on the case $s \leq 1$ and in fact our results will be proved in the case $s \in(0,1]$. See 


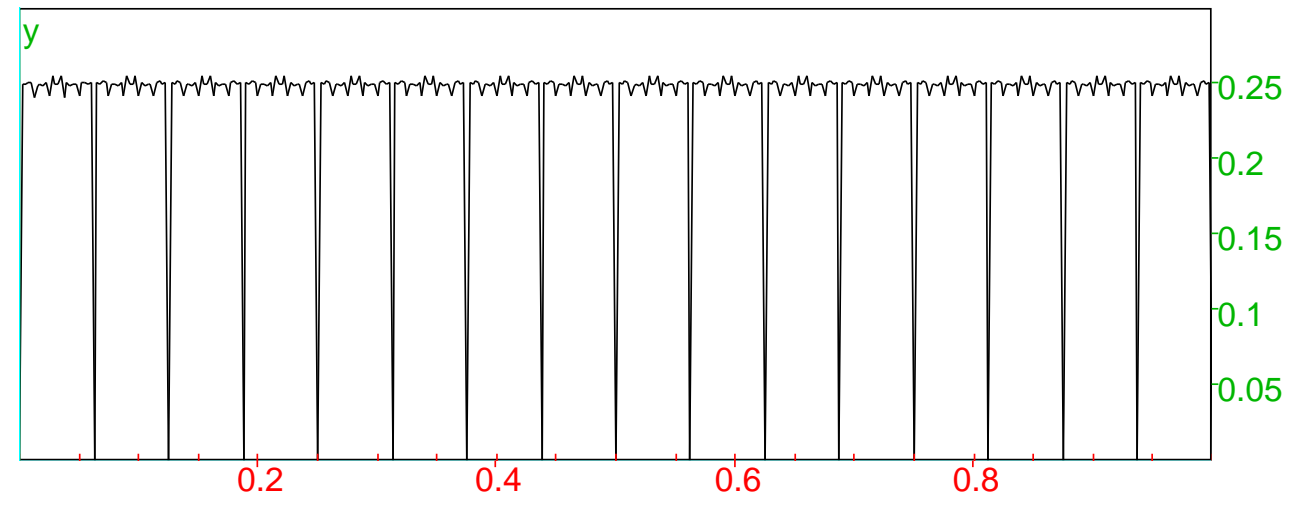

FiguRE 1. Graph of $F_{144}$

Theorem 2, $(v)$, for the case $s \leq 0$, which leads to results of a different nature that will not be investigated in depth. We set $H_{N}(s):=1+\frac{1}{2^{s}}+\cdots+\frac{1}{N^{s}}$ and note $H_{N}$ for $H_{N}(1)$. When $0 \leq s<1, H_{N}(s)=\frac{N^{1-s}}{1-s}+\mathcal{O}(1)$ whereas $H_{N}=\log (N)+\mathcal{O}(1)$ as $N \rightarrow+\infty$.

We will show that, given $s \in(0,1]$ and a rational number $\alpha, \frac{1}{H_{N}(s)} G_{s, N}(\alpha)$ converges to a rational number $<\frac{1}{4}$ that depends on the denominator of $\alpha$ and not on $s$. On the other hand, that sequence converges to $\frac{1}{4}$ for almost all irrational numbers $\alpha$, including for example the real irrational algebraic numbers, the numbers $e$ and $\pi$-only conjecturally for the latter when $s<1$. This does not seem to be the kind of result that helps to classify irrational numbers.

However, our most stricking results will concern the remainder

$$
\mathscr{G}_{s, N}(\alpha):=G_{s, N}(\alpha)-\frac{1}{4} H_{N}(s)=\sum_{n=1}^{N} \frac{1}{n^{s}}\left(F_{n}(\alpha)-\frac{1}{4}\right),
$$

when $\alpha$ is irrational. We will show that the sequence converges also almost surely as $N \rightarrow+\infty$ to a function $\mathscr{G}_{s}(\alpha)$ for $\left(^{2}\right)$ any given $s \in(0,1]$. (For the definition of the diophantine notions used from now on, see Section 1.3.) One of our results will be that the sequence $\left(\mathscr{G}_{1, N}(\alpha)\right)_{N \geq 1}$ converges for all irrational numbers with finite $m(\alpha)$ exponent and diverges for all rational numbers, leaving mainly open the question of convergence or divergence of $\left(\mathscr{G}_{1, N}(\alpha)\right)_{N \geq 1}$ for the rather sparse set of Liouville numbers. For $s \in(0,1)$, we will show that $\left(\mathscr{G}_{s, N}(\alpha)\right)_{N \geq 1}$ diverges for all rational numbers and that it converges, resp. diverges, for all irrational numbers $\alpha$ with $m(\alpha)<1+\frac{1}{1-s}$, resp. $m(\alpha)>2+\frac{4}{1-s}$.

Figure 2 illustrates the case $s=1$. It should be taken with precautions because our estimate for the speed of convergence of $\left(\mathscr{G}_{1, N}(\alpha)\right)_{N \geq 1}$ to $\mathscr{G}_{1}(\alpha)$ is not uniform on $\mathbb{R} \backslash \mathbb{Q}$. Nonetheless, it is quite surprising to observe that $\mathscr{G}_{1,200}\left(\frac{\sqrt{5}-1}{2}\right) \approx 0.2169$ seems to be very close to the maximum of $\mathscr{G}_{1,200}$. We don't know if in the limit, $\mathscr{G}_{1}\left(\frac{\sqrt{5}-1}{2}\right)$ coincides with

\footnotetext{
${ }^{2}$ Although this is not the point of view adopted in this paper, we can consider $\mathscr{G}_{s}(\alpha)$ as the Dirichlet series $\sum_{n=1}^{\infty} \frac{1}{n^{s}}\left(F_{n}(\alpha)-\frac{1}{4}\right)$ of the variable $s, \alpha$ being a parameter: our results will show the great dependence of the abscissa of convergence on the diophantine properties of $\alpha$, mainly $m(\alpha)$.
} 


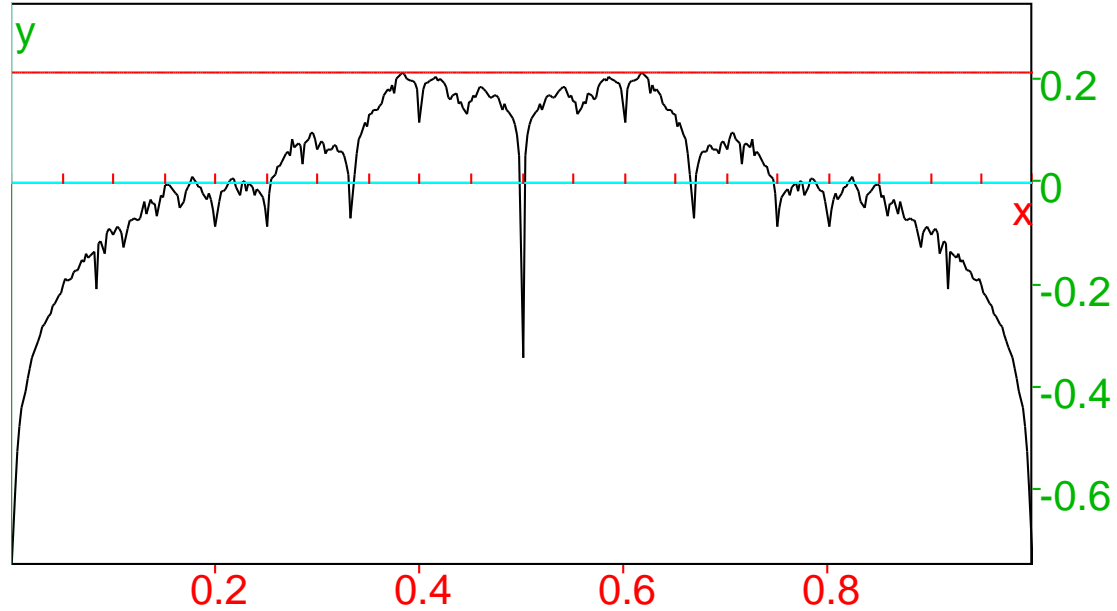

FiguRE 2. "Bear's pawprint" graph of $\mathscr{G}_{1,200}$ and the constant $\mathscr{G}_{1,200}\left(\frac{\sqrt{5}-1}{2}\right)$

the maximum of $\mathscr{G}_{1}$ but this is definitely an interesting problem to solve. The last section of the paper will present more graphs and numerical datas.

1.3. The results. We denote by $\left(p_{n} / q_{n}\right)_{n \geq 0}$ the sequence of convergents of an irrational number $\alpha$. Its partial quotients $\left(a_{n}\right)_{n \geq 0}$ are such that $q_{n+1}=a_{n+1} q_{n}+q_{n-1}$. Let us define a family $\mathscr{A}_{s}, s \in(0,1]$, of sets of irrational numbers $\alpha \in[0,1]$ such that, for $s \in(0,1)$,

$$
\sum_{m} \frac{q_{m+1}^{1-s}}{q_{m}}<\infty
$$

and, for $s=1$,

$$
\sum_{m} \frac{\log \left(\max \left(q_{m+1} / q_{m}, q_{m}\right)\right)}{q_{m}}<\infty .
$$

We recall that an irrational number $\alpha$ is said to have $a$ finite irrationality exponent $\mu(\alpha) \geq 2$ is there exists a constant $c(\alpha)>0$ such that $\left(^{3}\right)$

$$
\left|\alpha-\frac{p}{q}\right| \geq \frac{1}{c(\alpha) q^{\mu(\alpha)}}
$$

for all integers $p, q$ with $q \geq 1$. (In fact, $c(\alpha) \geq 1$ because of the case $q=1$ and $p=\lfloor\alpha\rfloor$.) We denote by $m(\alpha)$ the irrationality exponent of $\alpha$, defined as the infinimum of all possible $\mu(\alpha)$, regardless of the value of $c(\alpha)$. By definition, Liouville numbers are precisely those irrational numbers which don't have a finite irrationality exponent; they are not only irrational but also transcendental.

We first state a lemma, whose proof is postponed to Section 3.

\footnotetext{
${ }^{3}$ When we will talk about an irrationality exponent for an irrational number $\alpha$, one should understand a couple $(\mu(\alpha), c(\alpha))$.
} 
Lemma 1. (i) The set $\mathscr{A}_{1}$ contains all irrational numbers with a finite irrationality exponent. Some Liouville numbers belong to $\mathscr{A}_{1}$, some do not.

(ii) For any $s \in(0,1)$, the set $\mathscr{A}_{s}$ contains all irrational numbers with $m(\alpha)<1+\frac{1}{1-s}$ but no real number whose irrationality exponent $m(\alpha)$ is $>1+\frac{1}{1-s}$. In particular, it does not contain any Liouville number. Some irrational numbers with $m(\alpha)=1+\frac{1}{1-s}$ belong to $\mathscr{A}_{s}$, some do not.

(iii) The sets $\mathscr{A}_{s}$ all have measure 1.

Set

$$
\Phi_{s}(\alpha):=\sum_{n=1}^{\infty} \frac{1}{n^{s+1}} \sum_{m=1}^{n} \cos (2 m n \pi \alpha)=\sum_{n=1}^{\infty} \frac{\cos (\pi n(n+1) \alpha) \sin \left(\pi n^{2} \alpha\right)}{n^{s+1} \sin (\pi n \alpha)}
$$

and

$$
\Phi_{s, N}(\alpha):=\sum_{n=1}^{N} \frac{\cos (\pi n(n+1) \alpha) \sin \left(\pi n^{2} \alpha\right)}{n^{s+1} \sin (\pi n \alpha)}
$$

the $N$-th partial sum. We discard the case $s>1$ because the series trivially converges for any $\alpha$ (as is clear from the first expression for $\Phi_{s}(\alpha)$ ). The reason to consider $\Phi_{s}(\alpha)$ is due to the relation

$$
\mathscr{G}_{s, N}(\alpha)=-\frac{2}{\pi^{2}} \sum_{k=0}^{\infty} \frac{\Phi_{s, N}((2 k+1) \alpha)}{(2 k+1)^{2}},
$$

which will be proved later. Hence, $\Phi_{s, N}$ is a brick in the study of $\mathscr{G}_{s, N}$ and this explain why we study it first in Theorem 1 . Given some non-zero integers $a$ and $b$, we denote the greatest common divisor of $a$ and $b$ by $\operatorname{gcd}(a, b)$.

We can now state our main results.

Theorem 1. Let us fix $s \in(0,1]$.

(i) For any rational number $a / b$ with $\operatorname{gcd}(a, b)=1, b \geq 1$, we have

$$
\lim _{N \rightarrow+\infty} \frac{1}{H_{N}(s)} \Phi_{s, N}\left(\frac{a}{b}\right)=\frac{1}{b} .
$$

In particular,

$$
\lim _{N \rightarrow+\infty} \Phi_{s, N}\left(\frac{a}{b}\right)=+\infty .
$$

(ii) If $\alpha \in \mathscr{A}_{s}$, then the series $\Phi_{s}(\alpha)$ converges absolutely.

(iii) The sequence $\left(\Phi_{s, N}\right)_{N \geq 1}$ converges to $\Phi_{s}$ almost surely and in $L^{2}(0,1)$.

(iv) If $s \in(0,1)$ and if the irrational number $\alpha$ has an irrationality exponent $\mu(\alpha)>$ $2+\frac{4}{1-s}$, then for any $\varepsilon>0$,

$$
\limsup _{N \rightarrow+\infty} \frac{\Phi_{s, N}(\alpha)}{H_{N}(s)^{1-\frac{3-s}{\mu(\alpha)(1-s)}-\varepsilon}}=+\infty .
$$

This also holds if $\alpha$ is a Liouville number by setting $1 / \mu(\alpha)=0$. 
If $s=1$, there exists a dense set of Liouville numbers $\alpha$ such that, for any $\varepsilon>0$,

$$
\limsup _{N \rightarrow+\infty} \frac{\Phi_{1, N}(\alpha)}{H_{N}^{1-\varepsilon}}=+\infty \text {. }
$$

In all these cases, the sequence $\left(\Phi_{s, N}(\alpha)\right)_{N \geq 1}$ does not converge.

$(v)$ For any real numbers $\alpha$ and $s \leq 0$, the sequence $\left(\Phi_{s, N}(\alpha)\right)_{N \geq 1}$ does not converge.

Remarks 1. a) For $s \in(0,1)$, the result in $(i v)$ is probably not optimal and in particular one may expect the divergence of the series $\Phi_{s}(\alpha)$ for all $\alpha$ such that $m(\alpha)>1+\frac{1}{1-s}$. Nonetheless, for Liouville numbers (where one sets $1 / \mu(\alpha)=0$ ), it is essentially best possible, even when $s=1$, because $\left|\Phi_{s, N}(\alpha)\right| \leq H_{N}(s)$ for any real numbers $\alpha$ and $s$.

b) Item $(i v)$ is interesting because, together with $(i i)$, it shows that the convergence holds in case $s=1$ for a lot more irrational numbers (i.e. all but some Liouville numbers) than it does for other values of $s \in(0,1)$. The same dichotomy is also obtained in items (ii) and $(i v)$ of Theorem 2 below for the functions $\mathscr{G}_{s}$.

c) We will use the identity

$$
\int_{0}^{1} \Phi_{s}(\alpha)^{2} \mathrm{~d} \alpha=\frac{1}{2} \sum_{m=1}^{\infty} \sum_{n=1}^{\infty} \frac{\operatorname{gcd}(m, n)}{(m n)^{s+1}},
$$

which will be a consequence of the proof of (iii) where, in particular, the convergence of the double series will be proved.

d) Item $(v)$ is a consequence of the identity

$$
\limsup _{n \rightarrow+\infty} \frac{1}{n} \sum_{m=1}^{n} \cos (2 \pi m n \alpha)=\frac{L(\alpha)}{2 \pi} \sin \left(\frac{2 \pi}{L(\alpha)}\right) \neq 0,
$$

which will be proved in Lemma 6 .

The results of Theorem 1, as well as the methods of proof, will be useful to understand the behavior of $G_{s, N}(\alpha)$, which we now describe. We recall that $\mathscr{G}_{s, N}(\alpha)=G_{s, N}(\alpha)-\frac{1}{4} H_{N}(s)$.

Theorem 2. (i) Let us fix $s \in(0,1]$. For any rational number $a / b$ with $\operatorname{gcd}(a, b)=1$, $b \geq 1$, we have

$$
\begin{aligned}
\lim _{N \rightarrow+\infty} \frac{1}{H_{N}(s)} G_{s, N}\left(\frac{a}{b}\right) & =\frac{1}{4}-\frac{2}{\pi^{2}} \sum_{\substack{k=0 \\
\infty}}^{\infty} \frac{\operatorname{gcd}(b, 2 k+1)}{b(2 k+1)^{2}} \\
& =\frac{1}{4}-\frac{1}{4 b} \prod_{\substack{p \geq 3 \\
p \mid b}}\left(\frac{\left(p^{v_{p}(b)+1}-1\right)(p+1)-1}{p^{v_{p}(b)+2}}\right) .
\end{aligned}
$$

This limit is a rational number $<\frac{1}{4}$. In particular,

$$
\lim _{N \rightarrow+\infty} \mathscr{G}_{s, N}\left(\frac{a}{b}\right)=-\infty
$$


(ii) For any $s \in(0,1)$ and any $\alpha \in \mathscr{A}_{s}$ with $m(\alpha)<1+\frac{1}{1-s}$, or for $s=1$ and any $\alpha$ with finite $m(\alpha)$, we have

$$
\lim _{N \rightarrow+\infty} \mathscr{G}_{s, N}(\alpha)=-\frac{2}{\pi^{2}} \sum_{k=0}^{\infty} \frac{\Phi_{s}((2 k+1) \alpha)}{(2 k+1)^{2}}=: \mathscr{G}_{s}(\alpha),
$$

where the series $\mathscr{G}_{s}(\alpha)$ converges absolutely.

(iii) The sequence $\left(\mathscr{G}_{s, N}\right)_{N \geq 1}$ converges to $\mathscr{G}_{s}$ almost surely and in $L^{2}(0,1)$.

(iv) For any $s \in(0,1)$, if the irrational number $\alpha$ has an irrationality exponent $\mu(\alpha)>$ $2+\frac{4}{1-s}$ (in particular, if it is a Liouville number), then for any $\varepsilon>0$,

$$
\liminf _{N \rightarrow+\infty} \frac{\mathscr{G}_{s, N}(\alpha)}{H_{N}(s)^{1-\frac{3-s}{\mu(\alpha)(1-s)}-\varepsilon}}=-\infty .
$$

This also holds if $\alpha$ is a Liouville number by setting $1 / \mu(\alpha)=0$.

For $s=1$, there exists a dense set of Liouville numbers such that, for any $\varepsilon>0$,

$$
\liminf _{N \rightarrow+\infty} \frac{\mathscr{G}_{1, N}(\alpha)}{H_{N}^{1-\varepsilon}}=-\infty
$$

In all these cases, the sequence $\left(\mathscr{G}_{s, N}(\alpha)\right)_{N \geq 1}$ does not converge.

(v) For any real numbers $\alpha$ and $s \leq 0$, the sequence $\left(\mathscr{G}_{s, N}(\alpha)\right)_{N \geq 1}$ does not converge.

Remark 2. To appreciate these results, it is useful to have in mind the trivial bound

$$
\left|\mathscr{G}_{s, N}(\alpha)\right| \leq \frac{1}{4} H_{N}(s),
$$

which holds for any real numbers $\alpha$ and $s$.

We will also prove the following theorem, which is of independent interest. It provides examples of Fourier series that converge almost everywhere but at no rational point. For $s \in(0,1]$, we define $\mathscr{B}_{s}$ as the set of irrational numbers in $[0,1]$ such that $\sum_{n} q_{n+1} / q_{n}^{s+1}$ is convergent. This set is of measure 1 and contains all the irrational numbers with irrationality exponent $<s+2$ and no numbers with irrationality exponent $>s+2$; we always have $\mathscr{B}_{s} \subseteq \mathscr{A}_{s}$.

Theorem 3. Let us fix $s \in(0,1]$.

(i) The Fourier series of $\Phi_{s}$ is given by

$$
S\left(\Phi_{s}\right)(\alpha):=\sum_{k=1}^{\infty}\left(\sum_{n \mid k, n \geq \sqrt{k}} \frac{1}{n^{s+1}}\right) \cos (2 \pi k \alpha) .
$$

The series $S\left(\Phi_{s}\right)(\alpha)$ converges almost surely. More precisely, it is equal to $\Phi_{s}(\alpha)$ for all $\alpha \in \mathscr{B}_{s}$. It also converges to $\Phi_{s}$ in $L^{2}(0,1)$. 
(ii) The series $S\left(\Phi_{s}\right)$ converges for no rational number. More precisely, let $S_{s, N}$ denote the $N$-th partial sum of $S\left(\Phi_{s}\right)$. Then, for any rational number $a / b$ with $\operatorname{gcd}(a, b)=1$, $b \geq 1$, we have

$$
\lim _{N \rightarrow+\infty} \frac{1}{H_{\lfloor\sqrt{N}\rfloor}(s)} S_{s, N}\left(\frac{a}{b}\right)=\frac{2}{b(1+s)} .
$$

(iii) There exists a dense set of Liouville numbers on which $S\left(\Phi_{s}\right)$ does not converge.

The initial impulse to prove these results is given by the Fourier series of the function $\|\alpha\|$, which converges normally on $\mathbb{R}$ :

$$
\|\alpha\|=\frac{1}{4}-\frac{2}{\pi^{2}} \sum_{k=0}^{\infty} \frac{\cos (2(2 k+1) \pi \alpha)}{(2 k+1)^{2}} .
$$

Its form already explains (1.1).

We conclude this Introduction by mentioning related results. Setting

$$
\widehat{F}_{n}(\alpha):=\frac{1}{n} \sum_{m=1}^{n}\left(\{n m \alpha\}-\frac{1}{2}\right)^{2},
$$

the Fourier expansion

$$
\left(\{\alpha\}-\frac{1}{2}\right)^{2}=\frac{1}{12}-\frac{1}{\pi^{2}} \sum_{k=1}^{\infty} \frac{\cos (2 \pi k \alpha)}{k^{2}}
$$

implies that

$$
\sum_{n=1}^{N} \frac{1}{n^{s}}\left(\widehat{F}_{n}(\alpha)-\frac{1}{12}\right)=-\frac{1}{\pi^{2}} \sum_{k=1}^{\infty} \frac{\Phi_{s, N}(k \alpha)}{k^{2}} .
$$

Results very similar to those obtained for $F_{n}(\alpha)$ can be proved for $\widehat{F}_{n}(\alpha)$ and extremal properties around $\frac{\sqrt{5}-1}{2}$ and $\frac{3-\sqrt{5}}{2}$ can be observed as well.

It is also possible to partially generalise our results to the sequence $(\{j \alpha+\beta\})_{1 \leq j \leq n}$. For this, one starts from the identity

$$
\sum_{n=1}^{N} \frac{1}{n^{s}}\left(\frac{1}{n} \sum_{m=1}^{n}\|m n \alpha+n \beta\|-\frac{1}{4}\right)=-\frac{2}{\pi^{2}} \sum_{k=0}^{\infty} \frac{\Phi_{s, N}((2 k+1) \alpha, \beta)}{(2 k+1)^{2}},
$$

where

$$
\Phi_{s, N}(\alpha, \beta)=\sum_{n=1}^{N} \frac{\cos (\pi n(n \alpha+\alpha+2 \beta)) \sin \left(\pi n^{2} \alpha\right)}{n^{s+1} \sin (\pi n \alpha)} .
$$

By the methods of the paper, it is easy to prove that the convergence of $\Phi_{s, N}(\alpha, \beta)$ occurs at least for any $\alpha \in \mathscr{A}_{s}$ and any real number $\beta$; indeed, in the proofs of the above theorems, we will simply bound $\mid \cos (\pi n(n \alpha+\alpha) \mid$ by 1 (when $\beta=0)$ and it does not change anything if instead we bound $\mid \cos (\pi n(n \alpha+\alpha+2 \beta) \mid$ by 1 . However, it is no longer true that there is divergence at all rational numbers. For example, $\Phi_{1, N}\left(0, \frac{1}{2}\right)$ tends to $\log (1 / 2)$ as $N \rightarrow+\infty$. Furthermore, extremality properties around $\frac{\sqrt{5}-1}{2}$ and $\frac{3-\sqrt{5}}{2}$ seem to fade away when $\beta \neq 0$. 


\section{Properties of $\left(F_{n}(\alpha)\right)_{n \geq 1}$}

In this section, we prove a few results concerning the sequence $\left(F_{n}(\alpha)\right)_{n \geq 1}$. A more exhaustive study would be interesting.

Theorem 4. $(i)$ The sequence $\left(F_{n}\right)_{n \geq 1}$ converges to $\frac{1}{4}$ in $L^{2}(0,1)$.

(ii) For any rational number $a / b$ with $\operatorname{gcd}(a, b)=1$, we have

$$
F_{n}\left(\frac{a}{b}\right)=\frac{1}{4}-\frac{2}{\pi^{2}} \sum_{\substack{\ell=0 \\ b \mid n(2 \ell+1)}}^{\infty} \frac{1}{(2 \ell+1)^{2}}+\mathcal{O}\left(\frac{b}{n}\right),
$$

where the implicit constant is absolute. In particular,

$$
\liminf _{n \rightarrow+\infty} F_{n}\left(\frac{a}{b}\right)=0 \quad \text { and } \quad \limsup _{n \rightarrow+\infty} F_{n}\left(\frac{a}{b}\right) \leq \frac{1}{4} .
$$

(iii) For any irrational number $\alpha$ and any $\varepsilon>0$, there exist infinitely many $n$ such that $q_{n}\left\|q_{n} \alpha\right\| \leq \frac{1}{L(\alpha)-\varepsilon}$ and simultaneously

$$
F_{q_{n}}(\alpha)=\frac{q_{n}+1}{2}\left\|q_{n} \alpha\right\| .
$$

(iv) Set

$$
\lambda(\alpha):=\frac{2}{\pi^{2}} \sum_{k=0}^{\infty} \frac{1}{(2 k+1)^{2}} \cdot \frac{L((2 k+1) \alpha)}{2 \pi} \cdot \sin \left(\frac{2 \pi}{L((2 k+1) \alpha)}\right),
$$

which is $\leq \frac{1}{4}$ with equality if and only if $L(\alpha)=+\infty$. Then, for any irrational number $\alpha$, we have

$$
\frac{1}{4}-\lambda(\alpha) \leq \liminf _{n \rightarrow+\infty} F_{n}(\alpha) \leq \frac{1}{2 L(\alpha)}
$$

and

$$
\frac{1}{4} \leq \limsup _{n \rightarrow+\infty} F_{n}(\alpha) \leq \frac{1}{4}+\lambda(\alpha)
$$

where we also assume that $m(\alpha)$ is finite to get the lower bound $\frac{1}{4}$.

Remarks 3. a) Since the sequence $n \mapsto \sum_{b \mid n(2 \ell+1)}^{\infty} \frac{1}{(2 \ell+1)^{2}}$ is positive and periodic of period $b$, we see that $F_{n}(a / b)$ oscillates nearly periodically without converging. The $\lim \sup _{n} F_{n}(a / b)$ can be $<\frac{1}{4}$ but it can also be equal to $\frac{1}{4}$ (consider $F_{2 m+1}(a / b)$ when $b$ is even, for example).

b) For irrational numbers $\alpha$, we always have $\liminf _{n} F_{n}(\alpha)=\frac{1}{2 \sqrt{5}}<\frac{1}{4}$ and the liminf is 0 when $L(\alpha)=+\infty$, i.e. for almost all real numbers. Concerning the lim sup, numerical experiments suggest that

$$
\limsup _{n} F_{n}(\alpha)=\lim _{n \rightarrow+\infty} F_{2 q_{n}}(\alpha)>\frac{1}{4}
$$


if $\alpha$ is equivalent to $\frac{\sqrt{5}+1}{2}$. It seems plausible that $\lim _{\sup _{n}} F_{n}(\alpha)>\frac{1}{4}$ fo any irrational number $\alpha$.

c) Given $\alpha$, the value of $L((2 k+1) \alpha)$ is not a simple function of $L(\alpha)$ and it is not easy to compute $\lambda(\alpha)$. However, the bounds $\sqrt{5} \leq L((2 k+1) \alpha) \leq(2 k+1) L(\alpha)$ and the decreasing of $x \mapsto \sin (x) / x$ on $[0,2 \pi / \sqrt{5}]$ imply that

$$
0 \leq \lambda(\alpha) \leq \frac{L(\alpha)}{2 \pi^{3}} \sum_{k=0}^{\infty} \frac{1}{2 k+1} \cdot \sin \left(\frac{2 \pi}{(2 k+1) L(\alpha)}\right)
$$

For example, $\lambda\left(\frac{\sqrt{5}-1}{2}\right) \leq 0.06708$.

Proof of Theorem 4. We will use various properties of continued fractions in the the sequel. The reader is refered to Kintchine's classical book [13] on this subject.

( $i$ ) Using the Fourier expansion (1.2) of $\|\alpha\|$, we find that, for any real number $\alpha$,

$$
\begin{aligned}
F_{n}(\alpha)-\frac{1}{4} & =\frac{1}{n} \sum_{k=1}^{n}\left(\|k n \alpha\|-\frac{1}{4}\right) \\
& =-\frac{2}{\pi^{2} n} \sum_{k=1}^{n} \sum_{\ell=0}^{\infty} \frac{\cos (2(2 \ell+1) k n \pi \alpha)}{(2 \ell+1)^{2}} \\
& =-\frac{2}{\pi^{2} n} \sum_{\ell=0}^{\infty} \frac{1}{(2 \ell+1)^{2}} \sum_{k=1}^{n} \cos (2(2 \ell+1) k n \pi \alpha) \\
& =-\frac{2}{\pi^{2} n} \sum_{\ell=0}^{\infty} \frac{1}{(2 \ell+1)^{2}} \frac{\cos ((2 \ell+1) n(n+1) \pi \alpha) \sin \left((2 \ell+1) n^{2} \pi \alpha\right)}{\sin ((2 \ell+1) n \pi \alpha)}
\end{aligned}
$$

with standard conventions when $\sin ((2 \ell+1) n \pi \alpha)=0$.

In the sequel, $\|\cdot\|_{2}$ denotes the norm in $L^{2}(0,1)$, without any possibility of confusion with the distance to $\mathbb{Z}$ already denoted by $\|\cdot\|$. Set $u_{n}(\alpha):=\sum_{k=1}^{n} \cos (2 k n \pi \alpha)$. We claim that $\left\|u_{n}(\alpha)\right\|_{2}=\sqrt{n / 2}$. Indeed,

$$
\int_{0}^{1} u_{n}(\alpha)^{2} \mathrm{~d} \alpha=\sum_{k=1}^{n} \sum_{\ell=1}^{n} \int_{0}^{1} \cos (2 k n \pi \alpha) \cos (2 \ell n \pi \alpha) \mathrm{d} \alpha=\frac{1}{2} \sum_{1 \leq k=\ell \leq n} 1=\frac{n}{2}
$$

Moreover by 1-periodicity of $u_{n}(\alpha)$, we also have $\left\|u_{n}((2 \ell+1) \alpha)\right\|_{2}=\sqrt{n / 2}$ for any integer $\ell \geq 0$. 
Hence,

$$
\begin{aligned}
\left\|F_{n}(\alpha)-\frac{1}{4}\right\|_{2} & \leq \frac{2}{\pi^{2} n} \sum_{\ell=0}^{\infty} \frac{1}{(2 \ell+1)^{2}}\left\|u_{n}((2 \ell+1) \alpha)\right\|_{2} \\
& \leq \frac{2\left\|u_{n}(\alpha)\right\|_{2}}{\pi^{2} n} \sum_{\ell=0}^{\infty} \frac{1}{(2 \ell+1)^{2}} \\
& \leq \frac{1}{4 n}\left\|u_{n}(\alpha)\right\|_{2} \ll \frac{1}{\sqrt{n}} .
\end{aligned}
$$

It follows that $\left(F_{n}\right)_{n \geq 1}$ converges to $\frac{1}{4}$ in $L^{2}(0,1)$.

(ii) Let us fix a rational number $a / b$, with $\operatorname{gcd}(a, b)=1$ and $b \geq 1$; in particular, 0 is represented as $0 / 1$. We start again with the Fourier series (1.2):

$$
F_{n}\left(\frac{a}{b}\right)-\frac{1}{4}=-\frac{2}{\pi^{2}} \sum_{\ell=0}^{\infty} \frac{1}{(2 \ell+1)^{2}} \frac{1}{n} \sum_{k=1}^{n} \cos \left(2 \pi(2 \ell+1) k n \frac{a}{b}\right) .
$$

In order to use the periodicity of cos, we write $k=r b+j$ with $1 \leq j \leq b$ and $r \geq 0$, so that

$$
\begin{aligned}
\sum_{k=1}^{n} \cos \left(2(2 \ell+1) \pi k n \frac{a}{b}\right) & =\sum_{j=1}^{b} \sum_{\substack{r \geq 0 \\
r b+j \leq n}} \cos \left(2 \pi(2 \ell+1)(r b+j) n \frac{a}{b}\right) \\
& =\sum_{j=1}^{b} \cos \left(2 \pi(2 \ell+1) j n \frac{a}{b}\right) \sum_{\substack{r \geq 0 \\
r b+j \leq n}} 1 \\
& =\sum_{j=1}^{b} \cos \left(2 \pi(2 \ell+1) j n \frac{a}{b}\right)\left\lfloor\frac{n-j}{b}+1\right\rfloor \\
& =\frac{n}{b} \sum_{j=1}^{b} \cos \left(2 \pi(2 \ell+1) j n \frac{a}{b}\right)+\mathcal{O}(b),
\end{aligned}
$$

where the constant in the $\mathcal{O}$ is absolute.

Hence,

$$
\begin{aligned}
F_{n}\left(\frac{a}{b}\right)-\frac{1}{4} & =-\frac{2}{\pi^{2}} \sum_{\ell=0}^{\infty} \frac{1}{(2 \ell+1)^{2}} \frac{1}{b} \sum_{j=1}^{b} \cos \left(2 \pi(2 \ell+1) j n \frac{a}{b}\right)+\mathcal{O}\left(\frac{b}{n}\right) \\
& =-\frac{2}{\pi^{2}} \sum_{\substack{\ell=0 \\
b \mid n(2 \ell+1)}}^{\infty} \frac{1}{(2 \ell+1)^{2}}+\mathcal{O}\left(\frac{b}{n}\right)
\end{aligned}
$$


where we have used the fact that, for any integer $k$ and any rational $u / v$, with $\operatorname{gcd}(u, v)=1$ and $v \geq 1$,

$$
\sum_{j=1}^{v} \cos \left(2 \pi \frac{j k u}{v}\right)=\left\{\begin{array}{lll}
0 & \text { if } & v \nmid k \\
v & \text { if } & v \mid k
\end{array}\right.
$$

The estimates for the liminf and limsup follow from the two obvious facts:

$$
F_{b n}\left(\frac{a}{b}\right)=0
$$

and

$$
F_{n}\left(\frac{a}{b}\right) \leq \frac{1}{4}+\mathcal{O}\left(\frac{b}{n}\right)
$$

respectively.

(iii) By definition, $L(\alpha)=\limsup _{q \rightarrow+\infty} \frac{1}{q\|q \alpha\|}$, hence for any $\varepsilon$, there exist infinitely many positive integers $b_{n}$ (depending on $\alpha$ and $\varepsilon$ ) such that

$$
b_{n}\left\|b_{n} \alpha\right\| \leq \frac{1}{L(\alpha)-\varepsilon} .
$$

(Without loss of generality, we can even assume that $a_{n}:=\left\lfloor b_{n} \alpha\right\rceil$ and $b_{n}$ are coprime.) Since $L(\alpha) \geq \sqrt{5}$ for any irrational number $\alpha$, we can choose $\varepsilon$ small enough such that $L(\alpha)>2+\varepsilon$ (and thus $b_{n}\left\|b_{n} \alpha\right\|<\frac{1}{2}$, which implies that $a_{n} / b_{n}$ are convergents to $\alpha$ by a classical property of continued fractions).

Now, for any integer $k \in\left\{1,2, \ldots, b_{n}\right\}$, we have

$$
\left|k b_{n} \alpha-k a_{n}\right|=k\left|b_{n} \alpha-a_{n}\right| \leq \frac{k}{b_{n}(L(\alpha)-\varepsilon)} \leq \frac{1}{L(\alpha)-\varepsilon}<\frac{1}{2} .
$$

This forces that $k a_{n}=\left\lfloor k b_{n} \alpha\right\rceil$ and therefore

$$
F_{b_{n}}=\frac{1}{b_{n}} \sum_{k=1}^{b_{n}}\left\|b_{n} k \alpha\right\|=\frac{1}{b_{n}} \sum_{k=1}^{b_{n}}\left|k b_{n} \alpha-k b_{n}\right|=\frac{\left\|b_{n} \alpha\right\|}{b_{n}} \sum_{k=1}^{b_{n}} k=\frac{b_{n}+1}{2}\left\|b_{n} \alpha\right\|,
$$

as claimed.

(iv) For any $\varepsilon>0$ such that $L(\alpha)>2+\varepsilon$, we have

$$
\liminf _{n \rightarrow+\infty} F_{n}(\alpha) \leq \liminf _{n \rightarrow+\infty} F_{b_{n}}(\alpha)=\liminf _{n \rightarrow+\infty} \frac{b_{n}+1}{2}\left\|b_{n} \alpha\right\|=\frac{1}{2} \liminf _{n \rightarrow+\infty} b_{n}\left\|b_{n} \alpha\right\| \leq \frac{1}{2(L(\alpha)-\varepsilon)} .
$$

Since $\liminf _{n \rightarrow+\infty} F_{n}(\alpha)$ does not depend on $\varepsilon$, we get that

$$
\liminf _{n \rightarrow+\infty} F_{n}(\alpha) \leq \frac{1}{2 L(\alpha)}
$$


If we now assume that $\alpha$ has a finite irrationality exponent, then $\alpha \in \mathscr{A}_{1}$ and by Theorem $2,\left({ }^{4}\right)$ the series

$$
\sum_{n=1}^{\infty} \frac{F_{n}(\alpha)-\frac{1}{4}}{n}
$$

is convergent. Let us assume that $\limsup _{n} F_{n}(\alpha)<\frac{1}{4}$. Then there exists a constant $c>0$ such that $\frac{F_{n}(\alpha)-\frac{1}{4}}{n}<-\frac{c}{n}$ for all large enough $n$ and the above series cannot converge. This contradiction proves that

$$
\limsup _{n} F_{n}(\alpha) \geq \frac{1}{4}
$$

It remains to prove the two bounds involving $\lambda(\alpha)$. We have

$$
\left|F_{n}(\alpha)-\frac{1}{4}\right| \leq \frac{2}{\pi^{2}} \sum_{k=0}^{\infty} \frac{1}{(2 k+1)^{2}}\left|\frac{\cos ((2 k+1) n(n+1) \pi \alpha) \sin \left((2 k+1) n^{2} \pi \alpha\right)}{n \sin ((2 k+1) n \pi \alpha)}\right| .
$$

We will prove in Lemma 6 of Section 5.5 that, $\left({ }^{5}\right)$ for any $k$,

$$
\begin{aligned}
& \limsup _{n \rightarrow+\infty}\left|\frac{\cos ((2 k+1) n(n+1) \pi \alpha) \sin \left((2 k+1) n^{2} \pi \alpha\right)}{n \sin ((2 k+1) n \pi \alpha)}\right| \\
& \quad \leq \frac{L((2 k+1) \alpha)}{2 \pi} \cdot \sin \left(\frac{2 \pi}{L((2 k+1) \alpha)}\right) .
\end{aligned}
$$

Hence, by the reverse Fatou's Lemma, we obtain that

$$
\limsup _{n \rightarrow+\infty}\left|F_{n}(\alpha)-\frac{1}{4}\right| \leq \lambda(\alpha)
$$

It follows that

$$
\limsup _{n \rightarrow+\infty} F_{n}(\alpha) \leq \frac{1}{4}+\lambda(\alpha)
$$

and

$$
\liminf _{n \rightarrow+\infty} F_{n}(\alpha) \geq \frac{1}{4}-\lambda(\alpha)
$$

This finishes the proof of Theorem 4 .

\section{Proof of Lemma 1}

(i) Let $\alpha$ be an irrational number with a finite irrationality exponent $\mu(\alpha) \geq 2$, so that

$$
\left|\alpha-\frac{p}{q}\right| \geq \frac{1}{c(\alpha) q^{\mu(\alpha)}}
$$

\footnotetext{
${ }^{4}$ Item $(i v)$ of Theorem 4 will not be used in the proof of Theorem 2 .

${ }^{5}$ Again, item $(i v)$ will not be used in the proof of this lemma.
} 
for all integers $p, q$ with $q \geq 1$. In particular, if $p / q=p_{n} / q_{n}$ is the $n$-th convergent of $\alpha$, classical properties of continued fractions imply that

$$
\frac{1}{c(\alpha) q_{n}^{\mu(\alpha)}} \leq\left|\alpha-\frac{p_{n}}{q_{n}}\right| \leq \frac{1}{q_{n} q_{n+1}} .
$$

Thus, $q_{n+1} \leq c(\alpha) q_{n}^{\mu(\alpha)-1}$. Moreover, it is known that $q_{n} \geq 2^{(n-1) / 2}$ for all $n \geq 2$ and all irrational number $\alpha$.

It follows that both series

$$
\sum_{n} \frac{\log \left(\max \left(q_{n+1} / q_{n}, q_{n}\right)\right)}{q_{n}} \ll \sum_{n} \frac{\log \left(q_{n}\right)}{q_{n}}
$$

are convergent (at geometric rate).

The real number $\beta$ whose partial quotients are $a_{n}=2^{(n-1) !^{2}}$ is a Liouville number and the inequalities $2^{n !^{2}} q_{n} \leq q_{n+1} \leq 2^{n !^{2}+1} q_{n}$ ensure that $\beta \in \mathscr{A}_{1}$. On the other hand, the number $\kappa=\sum_{n=1}^{\infty} 1 / b_{n}$ with $b_{n+1}=2^{b_{n}}, b_{1}=1$, is also a Liouville number but it has infinitely many convergents such that $q_{n+1}=2^{q_{n}}$, so that $\kappa \notin \mathscr{A}_{1}$.

(ii) Similarly as above, we prove that

$$
\sum_{n} \frac{q_{n+1}^{1-s}}{q_{n}} \ll \sum_{n} \frac{1}{q_{n}^{1-(1-s)(\mu(\alpha)-1)}}
$$

and both series are convergent (at geometric rate) when $\mu(\alpha)<1+\frac{1}{1-s}$.

The convergence of the series $\sum_{m} q_{m+1}^{1-s} / q_{m}$ implies that $q_{m+1}=o\left(q_{m}^{\frac{1}{1-s}}\right)$ and this in turn implies that a member of $\mathcal{A}_{s}$ cannot have $m(\alpha)>1+\frac{1}{1-s}$, hence cannot be a Liouville number. Examples of continued fractions can be contructed that have exact irrationality exponent $1+\frac{1}{1-s}$ for which the series $\sum_{m} q_{m+1}^{1-s} / q_{m}$ converges or not.

(iii) Almost all real numbers have $m(\alpha)=2$. Hence, for any $s \in(0,1]$, almost all real numbers belong to $\mathscr{A}_{s}$.

\section{Some Diophantine estimates}

In this section, we will prove side results needed to prove Theorems 1 and 2 . They are interesting for themselves and that's why we prove them separetely. We have stated them completely explicitely because they will be used for numerical computation. But the value of the constants is not essential for the proofs of Theorem 1, 2 and 3 , for we only need to know on what they depend.

The sets $\mathscr{A}_{s}$ have been defined in the Introduction.

Proposition 1. (i) Let us fix $s \in(0,1]$. For any irrational number $\alpha \in \mathscr{A}_{s}$, the series

$$
\sum_{n=1}^{\infty} \frac{\left\|n^{2} \alpha\right\|}{n^{s+1}\|n \alpha\|}
$$

is convergent. In particular, it converges almost everywhere. 
(ii) For any irrational number $\alpha \in \mathscr{A}_{s}$, we have the following estimate for the speed of convergence: for any integer $m \geq 2$,

$$
\sum_{n=q_{m}}^{\infty} \frac{\left\|n^{2} \alpha\right\|}{n^{s+1}\|n \alpha\|} \leq\left\{\begin{array}{l}
\frac{2(1+\zeta(s+1))}{1-s} \sum_{k=m}^{\infty} \frac{1+q_{k+1}^{1-s}}{q_{k}} \text { if } 0<s<1 \\
2(1+\zeta(2)) \sum_{k=m}^{\infty} \frac{1+\log \left(\max \left(q_{k+1} / q_{k}, q_{k}\right)\right)}{q_{k}} \quad \text { if } s=1 .
\end{array}\right.
$$

(iii) If $\alpha \in \mathscr{A}_{s}$ (for some $s \in(0,1)$ ) and $\alpha$ has an irrationality exponent $\mu(\alpha)<1+\frac{1}{1-s}$, then for any $m \geq 6$, we have

$$
\begin{aligned}
\sum_{n=q_{m}}^{\infty} \frac{\left\|n^{2} \alpha\right\|}{n^{s+1}\|n \alpha\|} \leq \frac{2(1+\zeta(s+1))}{(1-s) q_{m}^{1-(\mu(\alpha)-1)(1-s)}}\left(3\left(1+c(\alpha)^{1-s}\right) \log \left(q_{m}\right)\right. & \\
& \left.+\frac{c(\alpha)^{1-s}}{1-\sqrt{2}^{(\mu(\alpha)-1)(1-s)-1}}\right) .
\end{aligned}
$$

(iv) If $\alpha \in \mathscr{A}_{1}$ has a finite irrationality exponent $\mu(\alpha)$, then for any $m \geq 6$, we have

$$
\sum_{n=q_{m}}^{\infty} \frac{\left\|n^{2} \alpha\right\|}{n^{2}\|n \alpha\|} \leq 2(1+\zeta(2))\left(3(1+\log c(\alpha)) \frac{\log \left(q_{m}\right)}{q_{m}}+5(\mu(\alpha)-1) \frac{\log \left(q_{m}\right)^{2}}{q_{m}}\right)
$$

Remark 4. The series (4.1) also converges for all real number $\alpha \in[0,1]$ when $s>1$, a result that follows immediately from Lemma 2. It is interesting to compare the upper bounds obtained in (ii) with the following ones, due to Kruse [14]: for any $s \geq 0$ and any irrationnal number $\alpha$, we have

$$
\sum_{n=q_{m}}^{q_{\ell}-1} \frac{1}{n^{s+1}\|n \alpha\|} \ll \sum_{k=m}^{\ell-1} \frac{q_{k+1}}{q_{k}^{s+1}} .
$$

The upper bound in (4.4) (which is optimal) displays the influence of our term $\left\|n^{2} \alpha\right\|$, in particular when $s=1$.

A few lemmas will be necessary for the proof.

Lemma 2. For any real number $\alpha \in[0,1]$ and any integer $n \geq 0$, we have

$$
\frac{\left\|n^{2} \alpha\right\|}{\|n \alpha\|} \leq \frac{n}{2\lfloor n\|n \alpha\|\rfloor+1}
$$

Proof. Let $j$ be an integer such that $0 \leq j \leq n$. The function $D_{n}(\alpha):=\frac{\|n \alpha\|}{\|\alpha\|}$ is increasing on $\left[\frac{j}{n}, \frac{j+1 / 2}{n}\right]$ and decreasing on $\left[\frac{j+1 / 2}{n}, \frac{j+1}{n}\right]$.

Since $D_{n}(j / n)=D_{n}((j+1) / n)=0$, we deduce that for all $\alpha \in\left[\frac{j}{n}, \frac{j+1}{n}[\right.$, we have

$$
0 \leq D_{n}(\alpha) \leq D_{n}\left(\frac{j+1 / 2}{n}\right)
$$




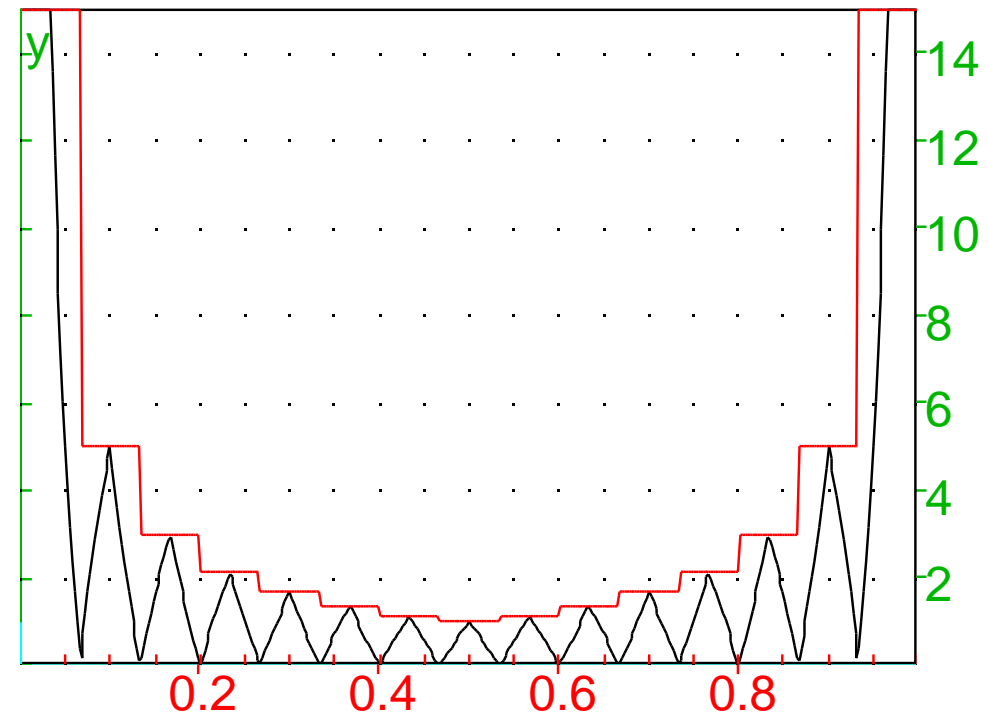

FiguRE 3. $D_{15}$ and its upper bound

If $\alpha \leq 1 / 2,\|\alpha\|=\alpha$ whereas if $\alpha>1 / 2,\|\alpha\|=1-\alpha$, whence after a moment of thought

$$
D_{n}\left(\frac{j+1 / 2}{n}\right)=\frac{n}{2\lfloor n\|\alpha\|\rfloor+1}
$$

for any $\alpha \in\left[\frac{j}{n}, \frac{j+1}{n}[\right.$.

We note that the right-hand side of the previous formula does not explicitly use the variable $j$. Therefore, we have shown that for any $\alpha \in[0,1]$, we have

$$
0 \leq D_{n}(\alpha) \leq \frac{n}{2\lfloor n\|\alpha\|\rfloor+1}
$$

See Figure 3 for an illustration of this upper bound. This formula enables to bound not only $D_{n}(\alpha)$ for $\alpha \in[0,1]$ but also for all $\alpha \in \mathbb{R}$ because $\|\alpha\|$ and $D_{n}(\alpha)$ are 1-periodic. Therefore, the upper bound (4.5) holds for any real number $\alpha$ and the lemma follows when replacing $\alpha$ by $n \alpha$.

Lemma 3. For any $\alpha \in[0,1]$ and any integer $N$ such that $q_{m} \leq N<q_{m+1}$, with $m \geq 2$, we have

$$
\sum_{k=q_{m}}^{N} \frac{\left\|k^{2} \alpha\right\|}{k^{s+1}|| k \alpha \|} \leq\left\{\begin{array}{l}
2(1+\zeta(2)) \cdot \frac{1+\log \left(\max \left(q_{m+1} / q_{m}, q_{m}\right)\right)}{q_{m}} \text { if } s=1 \\
\frac{2(1+\zeta(1+s))}{1-s} \cdot \frac{1+q_{m+1}^{1-s}}{q_{m}} \text { if } 0<s<1 .
\end{array}\right.
$$

Proof. By Lemma 2, it is enough to show that the same bound holds for the sum

$$
R_{N}:=\sum_{k=q_{m}}^{N} \frac{1}{k^{s}(2\lfloor k\|k \alpha\|\rfloor+1)} .
$$


Since

$$
0 \leq \frac{1}{2\lfloor k\|k \alpha\|\rfloor+1} \leq \frac{1}{k\|k \alpha\|}
$$

it is tempting to bound $R_{N}$ by

$$
\sum_{k=q_{m}}^{N} \frac{1}{k^{s+1}|| k \alpha \|}
$$

and then use Kruse's bound (4.4). But then we would lose the benefit of the inequalities

$$
0 \leq \frac{1}{2\lfloor k\|k \alpha\|\rfloor+1} \leq 1
$$

because the quantity $1 /\|k \alpha\|$ can take very large values. We will consider three cases: in the first one, (4.6) will be enough while for the remaining two, (4.7) will be used.

To study $R_{N}$, we adapt Kruse's ideas and cut the sum $R_{N}$ in three parts : $k \not \equiv 0, q_{m-1}\left[q_{m}\right]$, $k \equiv 0\left[q_{m}\right]$ and $k \equiv q_{m-1}\left[q_{m}\right]$. Remember that we suppose $q_{m} \leq N<q_{m+1}$, with $q_{m+1}=$ $a_{m+1} q_{m}+q_{m-1}$. Set $Q=\left\lfloor N / q_{m}\right\rfloor, r_{h}=q_{m}-1$ if $0 \leq h<Q$ and $r_{h}=N-Q q_{m}$ if $h=Q$. In particular, $0 \leq r_{h} \leq q_{m}-1$ and $Q<q_{m+1} / q_{m}$. The assumption that $n \geq 2$ ensures that $q_{m} \geq 2$ (a necessary assumption for Kruse's estimates) because $q_{m} \geq q_{2} \geq \sqrt{2}>1$.

- first step. We use (4.6):

$$
\begin{aligned}
\sum_{\substack{k=q_{m} \\
k \neq 0, q_{m-1}\left[q_{m}\right]}}^{N} \frac{1}{k^{s}} \frac{1}{2\lfloor k\|k \alpha\|\rfloor+1} & =\sum_{h=1}^{Q} \sum_{\substack{j=1 \\
j \neq q_{m-1}}}^{r_{h}} \frac{1}{\left(h q_{m}+j\right)^{s}\left(2\left\lfloor\left(h q_{m}+j\right)\left\|\left(h q_{m}+j\right) \alpha\right\|\right\rfloor+1\right)} \\
& \leq \sum_{h=1}^{Q} \sum_{\substack{j=1 \\
j \neq q_{m-1}}}^{r_{h}} \frac{1}{\left(h q_{m}+j\right)^{s+1}\left\|\left(h q_{m}+j\right) \alpha\right\|} .
\end{aligned}
$$

To deal with the sum over $j$, we then follow Kruse [14, pp. 240-241] verbatim to get (even when the sums are empty, in which case their values are 0$)$ :

$$
\begin{aligned}
\sum_{\substack{j=1 \\
j \neq q_{m-1}}}^{r_{h}} \frac{1}{\left(h q_{m}+j\right)^{s+1}\left\|\left(h q_{m}+j\right) \alpha\right\|} & \leq \frac{1}{\left(h q_{m}\right)^{s+1}} \sum_{\substack{j=1 \\
j \neq q_{m-1}}}^{r_{h}} \frac{1}{\left\|\left(h q_{m}+j\right) \alpha\right\|} \\
& \leq \frac{2}{\left(h q_{m}\right)^{s+1}} \sum_{k=1}^{r_{h}} \frac{1}{\frac{k}{q_{m}}} \\
& \leq \frac{2 q_{m}\left(1+\log \left(r_{h}+1\right)\right)}{\left(h q_{m}\right)^{s+1}} \\
& \leq 2 \frac{1+\log \left(q_{m}\right)}{q_{m}^{s} h^{s+1}} .
\end{aligned}
$$


Hence, finally,

$$
\begin{aligned}
\sum_{\substack{k=q_{m} \\
k \neq 0, q_{m-1}\left[q_{m}\right]}}^{N} \frac{1}{k^{s}} \frac{1}{2\lfloor k\|k \alpha\|\rfloor+1} & =\sum_{h=1}^{Q} \sum_{\substack{j=1 \\
j \neq q_{m-1}}}^{r_{h}} \frac{1}{\left(h q_{m}+j\right)^{s}\left(2\left\lfloor\left(h q_{m}+j\right)\left\|\left(h q_{m}+j\right) \alpha\right\|\right\rfloor+1\right)} \\
& \leq 2 \frac{1+\log \left(q_{m}\right)}{q_{m}^{s}} \sum_{h=1}^{Q} \frac{1}{h^{s+1}} \leq 2 \zeta(s+1) \frac{1+\log \left(q_{m}\right)}{q_{m}^{s}}
\end{aligned}
$$

- Second and third steps. We now use twice the inequality (4.7). We have

$$
\begin{aligned}
\sum_{\substack{k=q_{m} \\
k \equiv 0\left[q_{m}\right]}}^{N} \frac{1}{k^{s}} \frac{1}{2\lfloor k\|k \alpha\|\rfloor+1} & =\sum_{h=1}^{Q} \frac{1}{\left(h q_{m}\right)^{s}} \frac{1}{2\left\lfloor h q_{m}|| h q_{m} \alpha \|\right\rfloor+1} \leq \frac{1}{q_{m}^{s}} \sum_{h=1}^{Q} \frac{1}{h^{s}} \\
& \leq \begin{cases}\frac{1+\log \left(q_{m+1} / q_{m}\right)}{q_{m}} & \text { if } \quad s=1 \\
\frac{1}{1-s} \cdot \frac{1+q_{m+1}^{1-s}}{q_{m}} & \text { if } \quad 0<s<1 .\end{cases}
\end{aligned}
$$

Similarly, we have

$$
\begin{aligned}
& \sum_{\substack{k=q_{m} \\
k \equiv q_{m-1}\left[q_{m}\right]}}^{N} \frac{1}{k^{s}} \frac{1}{2\lfloor k \| k \alpha||\rfloor+1} \leq \sum_{h=1}^{a_{m+1}-1} \frac{1}{\left(h q_{m}+q_{m-1}\right)^{s}} \leq \frac{1}{q_{m}^{s}} \sum_{h=1}^{a_{m+1}} \frac{1}{h^{s}} \\
& \leq\left\{\begin{array}{l}
\frac{1+\log \left(q_{m+1} / q_{m}\right)}{q_{m}} \quad \text { if } \quad s=1 \\
\frac{1}{1-s} \cdot \frac{1+q_{m+1}^{1-s}}{q_{m}} \quad \text { if } \quad 0<s<1,
\end{array}\right.
\end{aligned}
$$

Adding together the three estimates (4.8), (4.9) and (4.10), whose sum is $R_{N}$, we immediately obtain the result in the case $s=1$. For $s \in(0,1)$, we get

$$
R_{N} \leq 2 \zeta(s+1) \frac{1+\log \left(q_{m}\right)}{q_{m}}+\frac{2}{1-s} \cdot \frac{1+q_{m+1}^{1-s}}{q_{m}}
$$

and the conclusion follows by the sequence of inequalities

$$
\log \left(q_{m}\right) \leq \log \left(q_{m+1}\right)=\frac{1}{1-s} \log \left(q_{m+1}^{1-s}\right) \leq \frac{1}{1-s} q_{m+1}^{1-s}
$$

We need one more lemma.

Lemma 4. Fix an integer $m \geq 6$ and an irrational number $\alpha$. 
For any $\omega>0$, we have

$$
\sum_{j=m}^{\infty} \frac{1}{q_{j}^{\omega}} \leq \frac{3 \log \left(q_{m}\right)}{q_{m}^{\omega}}+\frac{1}{1-(1 / 2)^{\omega / 2}} \cdot \frac{1}{q_{m}^{\omega}}
$$

For $\omega=1$, a better bound is

$$
\sum_{j=m}^{\infty} \frac{1}{q_{j}} \leq \frac{3 \log \left(q_{m}\right)}{q_{m}} .
$$

Furthermore,

$$
\sum_{j=m}^{\infty} \frac{\log \left(q_{j}\right)}{q_{j}} \leq \frac{5 \log \left(q_{m}\right)^{2}}{q_{m}} .
$$

Remark 5. Slightly better bounds will be given during the proof but they are more complicated and we don't need them.

Proof. Below, $\log _{2}(x):=\log (x) / \log (2)$. We recall that $q_{j} \geq 2^{(j-1) / 2}$ for any $j \geq 1$. For simplicity, we set $\delta_{m}=\left\lceil 2 \log _{2}\left(q_{m}\right)\right\rceil \geq m$. We have

$$
\begin{aligned}
\sum_{j=m}^{\infty} \frac{1}{q_{j}^{\omega}} & =\sum_{j=m}^{\delta_{m}} \frac{1}{q_{j}^{\omega}}+\sum_{j=\delta_{m}+1}^{\infty} \frac{1}{q_{j}^{\omega}} \\
& \leq \frac{\delta_{m}-m+1}{q_{m}^{\omega}}+\sum_{j=\delta_{m}+1}^{\infty} \frac{1}{2^{\omega(j-1) / 2}} \\
& \leq \frac{2 \log _{2}\left(q_{m}\right)-m+2}{q_{m}^{\omega}}+\frac{1}{1-(1 / 2)^{\omega / 2}} \cdot \frac{1}{2^{\omega \delta_{m} / 2}} \\
& \leq \frac{3 \log \left(q_{m}\right)-m+2}{q_{m}^{\omega}}+\frac{1}{1-(1 / 2)^{\omega / 2}} \cdot \frac{1}{q_{m}^{\omega}} .
\end{aligned}
$$

This gives (4.11) because $-m+2 \leq 0$.

If $\omega=1$, we remark that, for $m \geq 6,-m+2+\frac{1}{1-(1 / 2)^{1 / 2}}<0$ so that

$$
\frac{3 \log \left(q_{m}\right)-m+2}{q_{m}}+\frac{1}{1-(1 / 2)^{1 / 2}} \cdot \frac{1}{q_{m}} \leq \frac{3 \log \left(q_{m}\right)}{q_{m}}
$$

which gives (4.12).

We now prove (4.13). We set $\kappa_{m}=\left\lceil 3 \log _{2}\left(q_{m}\right)\right\rceil \geq m$. We also define $\varepsilon_{m}$ as the unique positive real number such that $\left(1 / \varepsilon_{m}\right)^{1 / \varepsilon_{m}}=q_{\kappa_{m}+1}$. In terms of Lambert function $W$, we have

$$
\varepsilon_{m}=\frac{W\left(\log \left(q_{\kappa_{m}+1}\right)\right)}{\log \left(q_{\kappa_{m}+1}\right)} .
$$

(The function $W$ is defined on $(0, \infty)$ by the implicit equation $x=W(x) e^{W(x)}$; see [6] for its properties.) Since $\kappa_{m} \geq m \geq 6$, then $q_{\kappa_{m}+1} \geq q_{7} \geq 2^{3}$; hence $0<\varepsilon_{m} \leq \frac{W(\log (8))}{\log (8)} \approx 0.41868$. 
It follows that $2^{-\kappa_{m}\left(1-\varepsilon_{m}\right) / 2} \leq 2^{-\kappa_{m} / 3} \leq 1 / q_{m}$ and that $0<\frac{1}{1-(1 / 2)^{\left(1-\varepsilon_{m}\right) / 2}}<5.5$. These bounds are used below.

The reason for introducing $\varepsilon_{m}$ is that $\log (x) \leq x^{\varepsilon_{m}}$ for any $x \geq\left(1 / \varepsilon_{m}\right)^{1 / \varepsilon_{m}}=q_{\kappa_{m}+1}$. We now make the same kind of computations as above:

$$
\begin{aligned}
\sum_{j=m}^{\infty} \frac{\log \left(q_{j}\right)}{q_{j}} & =\sum_{j=m}^{\kappa_{m}} \frac{\log \left(q_{j}\right)}{q_{j}}+\sum_{j=\kappa_{m}+1}^{\infty} \frac{\log \left(q_{j}\right)}{q_{j}} \leq \sum_{j=m}^{\kappa_{m}} \frac{\log \left(q_{j}\right)}{q_{j}}+\sum_{j=\kappa_{m}+1}^{\infty} \frac{q_{j}^{\varepsilon_{m}}}{q_{j}} \\
& \leq \frac{\kappa_{m}-m+1}{q_{m}} \log \left(q_{m}\right)+\sum_{j=\kappa_{m}+1}^{\infty} \frac{1}{2^{(j-1)\left(1-\varepsilon_{m}\right) / 2}} \\
& \leq \frac{3 \log _{2}\left(q_{m}\right)-m+2}{q_{m}} \log \left(q_{m}\right)+\frac{1}{1-(1 / 2)^{\left(1-\varepsilon_{m}\right) / 2}} \cdot \frac{1}{2^{\kappa_{m}\left(1-\varepsilon_{m}\right) / 2}} \\
& \leq \frac{5 \log \left(q_{m}\right)^{2}}{q_{m}}-\frac{(m-2) \log \left(q_{m}\right)}{q_{m}}+\frac{5.5}{q_{m}} \leq \frac{5 \log \left(q_{m}\right)^{2}}{q_{m}}
\end{aligned}
$$

where in the last inequality, we used the fact that $(m-2) \log \left(q_{m}\right)-5.5 \geq 4 \log \left(q_{6}\right)-5.5 \geq 0$.

This completes the proof of the lemma.

Proof of Proposition 1. Let us assume that $s=1$ and fix $\alpha \in \mathscr{A}_{1}$. By Lemma 3, for any integers $n, m$ such that $2 \leq n<m$, we have

$$
\sum_{k=q_{n}}^{q_{m}-1} \frac{\left\|k^{2} \alpha\right\|}{k^{2}\|k \alpha\|}=\sum_{j=n}^{m-1} \sum_{k=q_{j}}^{q_{j+1}-1} \frac{\left\|k^{2} \alpha\right\|}{k^{2}\|k \alpha\|} \leq 2(1+\zeta(2)) \sum_{j=n}^{m-1} \frac{1+\log \left(\max \left(q_{j+1} / q_{j}, q_{j}\right)\right)}{q_{j}} .
$$

Since both series converges when $m \rightarrow+\infty$, we deduce that

$$
\sum_{k=q_{n}}^{\infty} \frac{\left\|k^{2} \alpha\right\|}{k^{2}\|k \alpha\|} \leq 2(1+\zeta(2)) \sum_{j=n}^{\infty} \frac{1+\log \left(\max \left(q_{j+1} / q_{j}, q_{j}\right)\right)}{q_{j}}
$$

This proves $(i)$ and $(i i)$ in the case $s=1$. The proof can immediately be adapted to the case $0<s<1$.

Let us prove (iii). Consider $\alpha \in \mathscr{A}_{s}$ with an irrationality exponent $\mu:=\mu(\alpha)<1+\frac{1}{1-s}$, with the associated constant $c(\alpha) \geq 1$. We have already shown that $q_{j+1} \leq c(\alpha) q_{j}^{\mu-1}$. Hence,

It also follows that

$$
\frac{q_{j+1}^{1-s}}{q_{j}} \leq c(\alpha)^{1-s} \frac{1}{q_{j}^{1-(\mu-1)(1-s)}}
$$

$$
\sum_{j=m}^{\infty} \frac{q_{j+1}^{1-s}}{q_{j}} \leq c(\alpha)^{1-s} \sum_{j=m}^{\infty} \frac{1}{q_{j}^{1-(\mu-1)(1-s)}}
$$

For $m \geq 6$, we now use the bound (4.11) with $\omega=1-(\mu-1)(1-s)$ together with the bound (4.12) on the right hand side of the inequality in ( $i i)$. This gives (4.2). 
It remains to prove $(i v)$. If $\alpha \in \mathscr{A}_{1}$ has a finite irrationality exponent $\mu(\alpha)$, we have $q_{j+1} \leq c(\alpha) q_{j}^{\mu-1}$. Hence $\log \left(q_{j+1}\right) \leq(\mu(\alpha)-1) \log \left(q_{j}\right)+\log c(\alpha)$. It follows that

$$
\sum_{k=q_{m}}^{\infty} \frac{\left\|k^{2} \alpha\right\|}{k^{2}\|k \alpha\|} \leq 2(1+\zeta(2))\left((1+\log c(\alpha)) \sum_{j=m}^{\infty} \frac{1}{q_{j}}+(\mu(\alpha)-1) \sum_{j=m}^{\infty} \frac{\log \left(q_{j}\right)}{q_{j}}\right) .
$$

Eq. (4.3) follows by means of (4.12) and (4.13) when $m \geq 6$.

\section{Proof of TheOREM 1}

5.1. Proof of $(i)$. We fix a rational $a / b$ such that $\operatorname{gcd}(a, b)=1$ and $b \geq 1$. We have

$$
\Phi_{s, N}\left(\frac{a}{b}\right)=\sum_{n=1}^{N} \frac{1}{n^{s+1}} \sum_{m=1}^{n} \cos \left(2 m n \pi \frac{a}{b}\right)
$$

As in the proof of Theorem 4, $(i i)$, we write $m=r b+j$ with $1 \leq j \leq b$ and $r \geq 0$ and get

$$
\Phi_{s, N}\left(\frac{a}{b}\right)=\frac{1}{b} \sum_{j=1}^{b} \sum_{n=1}^{N} \frac{1}{n^{s}} \cos \left(2 \pi j n \frac{a}{b}\right)+\mathcal{O}\left(b H_{N}(s+1)\right),
$$

where the implicit constant is absolute. We treat similarly the sum over $n$ to get

$$
\Phi_{s, N}\left(\frac{a}{b}\right)=\frac{1}{b} \sum_{j=1}^{b} \sum_{k=1}^{b} \cos \left(2 \pi j k \frac{a}{b}\right) \sum_{\substack{n \leq N \\ n \equiv k[b]}} \frac{1}{n^{s}}+\mathcal{O}(b \zeta(s+1)) .
$$

Hence, since

$$
\sum_{\substack{n \leq N \\ n \equiv k[b]}} \frac{1}{n^{s}}=\frac{1}{b} H_{N}(s)+\mathcal{O}(1)
$$

where the constant depends at most on $s$, we deduce that

$$
\Phi_{s, N}\left(\frac{a}{b}\right)=H_{N}(s) \frac{1}{b^{2}} \sum_{j=1}^{b} \sum_{k=1}^{b} \cos \left(2 \pi j k \frac{a}{b}\right)+\mathcal{O}(b),
$$

where the constant depends at most on $s$.

It remain to compute

$$
\frac{1}{b^{2}} \sum_{j=1}^{b} \sum_{k=1}^{b} \cos \left(2 \pi j k \frac{a}{b}\right) .
$$

We do this in a slightly more general form that will be used later.

Lemma 5. For any rational number $u / v$ not necessarily reduced, with $v \geq 1$, we have

$$
\frac{1}{v} \sum_{j=1}^{v} \sum_{k=1}^{v} \cos \left(2 \pi j k \frac{u}{v}\right)=\operatorname{gcd}(u, v) .
$$


Proof. We denote by $S$ the double sum to be computed. The inner sum over $k$ equals $v$ if $v$ divides $j u$ and 0 otherwise. Thus,

$$
S=\sum_{\substack{j=1 \\ v \mid j u}}^{v} 1=\sum_{\substack{j=1, \ldots, v \\ \frac{v}{\operatorname{gcd}(u, v)} \mid j, \frac{u}{\operatorname{gcd}(u, v)}}} 1=\sum_{\substack{j=1, \ldots, v \\ v \\ \operatorname{gcd}(u, v)}} 1=\sum_{j=1}^{\operatorname{gcd}(u, v)} 1=\operatorname{gcd}(u, v) .
$$

Applying the lemma with $u=a$ and $v=b$ (which are coprime), we obtain that

$$
\Phi_{s, N}\left(\frac{a}{b}\right)=\frac{1}{b} H_{N}(s)+\mathcal{O}(b)
$$

(the constant depends on $s$ ), which proves the claim, in an even more precise form that will be used later.

5.2. Proof of $(i i)$. We remark that

$$
\left|\frac{\cos (\pi n(n+1) \alpha) \sin \left(\pi n^{2} \alpha\right)}{\sin (\pi n \alpha)}\right| \leq \frac{\left|\sin \left(\pi n^{2} \alpha\right)\right|}{|\sin (\pi n \alpha)|}=\frac{\sin \left(\pi\left\|n^{2} \alpha\right\|\right)}{\sin (\pi \| n \alpha||)} \leq \frac{\pi}{2} \frac{\left\|n^{2} \alpha\right\|}{\|n \alpha\|}
$$

(We have used the inequalities $2 x \leq \sin (\pi x) \leq \pi x$, which hold for any $x$ such that $0 \leq$ $x \leq 1 / 2$.)

By definition of $\Phi_{s, N}(\alpha)$, we deduce that

$$
\left|\Phi_{s, N}(\alpha)\right| \leq \sum_{n=1}^{N}\left|\frac{\cos (\pi n(n+1) \alpha) \sin \left(\pi n^{2} \alpha\right)}{n^{s+1} \sin (\pi n \alpha)}\right| \leq \frac{\pi}{2} \sum_{n=1}^{N} \frac{\left\|n^{2} \alpha\right\|}{n^{s+1}|| n \alpha \|}
$$

We can now use Proposition $1,(i)$, to conclude that both series converge for any $\alpha \in \mathscr{A}_{s}$.

5.3. Proof of $(i i i)$. - Almost sure convergence of $\Phi_{s, N}$. We have proved that $\Phi_{s, N}(\alpha)$ converges to $\Phi_{s}(\alpha)$ for all $\alpha \in \mathscr{A}_{s}$ and moreover, that $\mathscr{A}_{s}$ is of measure 1.

- Convergence of $\Phi_{s, N}$ to $\Phi_{s}$ in $L^{2}(0,1)$. 
Firstly, let us show that $\left(\Phi_{s, N}\right)_{n \geq 1}$ converges in $L^{2}$. For this, it is enough to show that the sequence is Cauchy. For any integers $N \geq M \geq 1$, we have

$$
\begin{aligned}
\int_{0}^{1}\left(\Phi_{s, N}(\alpha)\right. & \left.-\Phi_{s, M}(\alpha)\right)^{2} \mathrm{~d} \alpha \\
& =\sum_{m=M+1}^{N} \sum_{n=M+1}^{N} \frac{1}{(m n)^{s+1}} \sum_{k=1}^{m} \sum_{\ell=1}^{n} \int_{0}^{1} \cos (2 k m \pi \alpha) \cos (2 \ell n \pi \alpha) \mathrm{d} \alpha \\
& =\frac{1}{2} \sum_{m=M+1}^{N} \sum_{n=M+1}^{N} \frac{1}{(m n)^{s+1}} \sum_{\substack{1 \leq k \leq m, 1 \leq \ell \leq n \\
\ell m=k n}} 1 \\
& =\frac{1}{2} \sum_{m=M+1}^{N} \sum_{n=M+1}^{N} \frac{1}{(m n)^{s+1}} \sum_{\substack{1 \leq \ell \leq n \\
n \mid \ell m}} 1 \\
& =\frac{1}{2} \sum_{m=M+1}^{N} \sum_{n=M+1}^{N} \frac{\operatorname{gcd}(m, n)}{(m n)^{s+1}}
\end{aligned}
$$

(We use an identity obtain during the proof of Lemma 5 for the last equality.) For any integer $m \geq 1$, the Dirichlet series with positive terms $A_{m}(s+1):=\sum_{n=1}^{\infty} \frac{\operatorname{gcd}(m, n)}{n^{s+1}}$ is convergent and thus

$$
\int_{0}^{1}\left(\Phi_{s, N}(\alpha)-\Phi_{s, M}(\alpha)\right)^{2} \mathrm{~d} \alpha \leq \frac{1}{2} \sum_{m=M+1}^{N} \frac{A_{m}(s+1)}{m^{s+1}} .
$$

It remains to prove that the series with term $A_{m}(s+1) / m^{s+1}$ converges. For this, we proceed as follows. The arithmetic function $n \mapsto \operatorname{gcd}(m, n)$ is multiplicative and bounded by $m$. Therefore, $A_{m}(t)$ converges for any $t$ such that $\operatorname{Re}(t)>1$ and we have

$$
\begin{aligned}
A_{m}(t) & =\prod_{p}\left(\sum_{k=0}^{\infty} \frac{a\left(p^{k}\right)}{p^{k t}}\right) \\
& =\prod_{p \mid m}\left(\sum_{k=0}^{v_{p}(m)} \frac{p^{k}}{p^{k t}}+\sum_{k=v_{p}(m)+1}^{\infty} \frac{p^{v_{p}(m)}}{p^{k t}}\right) \cdot \prod_{p \nmid m}\left(\sum_{k=0}^{\infty} \frac{1}{p^{k t}}\right) \\
& =\zeta(t) \prod_{p \mid m}\left(\frac{1}{p^{(t-1) v_{p}(m)}}\left(\frac{p^{(t-1)\left(v_{p}(m)+1\right)}-1}{p^{t-1}-1}-\frac{1}{p^{t}-1}\right)\left(1-\frac{1}{p^{t}}\right)\right) .
\end{aligned}
$$

In particular, substituting $s+1$ for $t$ with $s>0$, we obtain the bound

$$
A_{m}(s+1) \ll \prod_{p \mid m}\left(1+\frac{1}{p^{s}}\right),
$$


where the implicit constant depends on $s$. Now, for any $s>0$, we have

$$
\prod_{p \mid m}\left(1+\frac{1}{p^{s}}\right)=\sum_{d \mid m} \frac{|\mu(d)|}{d^{s}} \leq \sum_{d \mid m} 1 \leq e^{\mathcal{O}(\log (m) / \log \log (m))}
$$

for some absolute constant (Extremal order of $\sum_{d \mid m} 1$, in Tenenbaum [21, p. 84]). Hence, we have proved that

$$
\left|\frac{A_{m}(s+1)}{m^{s+1}}\right| \ll \frac{e^{\mathcal{O}(\log (m) / \log \log (m))}}{m^{s+1}},
$$

where the right-hand side is the term of a convergent series, as was to be proved.

Therefore, $\left\|\Phi_{s, N}-\Phi_{s, M}\right\|_{2}$ tends to 0 when $N \geq M \rightarrow+\infty$, so that the sequence $\left(\Phi_{s, N}\right)_{N \geq 0}$ converges in $L^{2}(0,1)$ to a certain function $\widehat{\Phi}_{s}$. By a classical property, we deduce the existence of a subsequence $\left(\Phi_{N_{k}, s}\right)_{k}$ such that $\Phi_{N_{k}, s} \rightarrow \widehat{\Phi}_{s}$ almost surely, which implies that $\Phi_{s}=\widehat{\Phi}_{s}$ almost surely.

5.4. Proof of $(i v)$. Given some irrational number $\alpha$ and $s \in(0,1]$, that will be further specified later, we consider a sequence of coprime rational numbers $\left(a_{m} / b_{m}\right)_{m}$ that converges to $\alpha$.

By the mean value theorem, we have

$$
\begin{aligned}
\left|\Phi_{s, N}(\alpha)-\Phi_{s, N}\left(\frac{a_{m}}{b_{m}}\right)\right| & \leq \sum_{n=1}^{N} \frac{1}{n^{s+1}} \sum_{k=1}^{n}\left|\cos (2 \pi k n \alpha)-\cos \left(2 \pi k n \frac{a_{m}}{b_{m}}\right)\right| \\
& \leq \sum_{n=1}^{N} \frac{1}{n^{s+1}} \sum_{k=1}^{n}\left|2 \pi k n \alpha-2 \pi k n \frac{a_{m}}{b_{m}}\right| \\
& \leq 2 \pi\left|\alpha-\frac{a_{m}}{b_{m}}\right| \cdot \sum_{n=1}^{N} \frac{1}{n^{s}} \sum_{k=1}^{n} k \\
& \ll N^{3-s}\left|\alpha-\frac{a_{m}}{b_{m}}\right|,
\end{aligned}
$$

where the implicit constant depends only on $s$. Hence,

$$
\begin{aligned}
\Phi_{s, N}(\alpha) & =\Phi_{s, N}\left(\frac{a_{m}}{b_{m}}\right)+\mathcal{O}\left(N^{3-s}\left|\alpha-\frac{a_{m}}{b_{m}}\right|\right) \\
& =\frac{H_{N}(s)}{b_{m}}+\mathcal{O}\left(b_{m}\right)+\mathcal{O}\left(N^{3-s}\left|\alpha-\frac{a_{m}}{b_{m}}\right|\right),
\end{aligned}
$$

by (5.1) and where the implicit constants depend on $s$. We now distinguish the case $s=1$ from the case $s \in(0,1)$.

- Case $s=1$. Since $H_{N}=\log (N)+\mathcal{O}(1)$, Eq (5.4) becomes

$$
\Phi_{s, N}(\alpha)=\frac{\log (N)}{b_{m}}+\mathcal{O}\left(b_{m}\right)+\mathcal{O}\left(N^{2}\left|\alpha-\frac{a_{m}}{b_{m}}\right|\right) .
$$


Let us now assume that $\alpha$ is such that

$$
0<\left|\alpha-\frac{a_{m}}{b_{m}}\right| \leq \frac{1}{e^{\delta_{m} b_{m}^{2}}}
$$

where $\delta_{m}$ is some function tending to $+\infty$ with $m$. We can take for example $\alpha=\sum_{n \geq 1} 1 / b_{n}$ avec $b_{n+1}=2^{b_{n}^{3}}$ et $b_{1}=1$ : for any $m \geq 1$, we have

$$
\sum_{n=1}^{m} \frac{1}{b_{n}}=: \frac{a_{m}}{b_{m}}
$$

and obviously $\left(a_{m}, b_{m}\right)=1$ because $a_{m}$ is odd and $b_{m}$ is a pure power of 2 . Of course, the diophantine condition (5.6) implies that $\alpha$ is a Liouville number.

We choose $N=N_{m}=\left\lfloor e^{\frac{1}{2} \delta_{m} b_{m}^{2}}\right\rfloor$, so that (5.5) becomes

$$
\Phi_{1, N_{m}}(\alpha)=\frac{1}{2} \delta_{m} b_{m}+\mathcal{O}\left(b_{m}\right)+\mathcal{O}(1)
$$

Then, it follows that

$$
\limsup _{N \rightarrow+\infty} \Phi_{1, N}(\alpha)=+\infty
$$

In fact, (5.7) even implies that

$$
\Phi_{1, N_{m}}(\alpha) \gg \sqrt{\delta_{m} \log \left(N_{m}\right)},
$$

with $N_{m} \approx e^{\frac{1}{2} \delta_{m} b_{m}^{2}}$. We can choose $\delta_{m}$ much larger than $b_{m}$, and have $b_{m}=\mathcal{O}\left(\log \left(N_{m}\right)^{\varepsilon}\right)$ for any given $\varepsilon \in(0,1)$. Therefore, there exist infinitely many choices for the sequence $\left(\delta_{m}\right)_{m}$ (corresponding to infinitely many $\alpha$ ) such that $\delta_{m} \gg \log \left(N_{m}\right) / b_{m}^{2} \gg \log \left(N_{m}\right)^{1-2 \varepsilon}$, in which case we have

$$
\limsup _{N \rightarrow+\infty} \frac{\Phi_{1, N}(\alpha)}{\log (N)^{1-\varepsilon}}=+\infty
$$

as claimed. Clearly, we can make this contruction for a dense set of Liouville numbers.

- Case $s \in(0,1)$. Since $H_{N}(s)=\frac{N^{1-s}}{1-s}+\mathcal{O}(1)$, Eq (5.4) becomes

$$
\Phi_{s, N}(\alpha)=\frac{N^{1-s}}{(1-s) b_{m}}+\mathcal{O}\left(b_{m}\right)+\mathcal{O}\left(N^{3-s}\left|\alpha-\frac{a_{m}}{b_{m}}\right|\right) .
$$

Let us assume for the moment that $\alpha$ is not a Liouville number and is such that

$$
0<\left|\alpha-\frac{a_{m}}{b_{m}}\right| \leq \frac{1}{b_{m}^{\mu}}
$$

where $\left(a_{m}, b_{m}\right)=1$, for some $\mu>2+\frac{4}{1-s}$, which implies that the irrationality exponent of $\alpha$ is $\geq 2+\frac{4}{1-s}$.

We choose $N=N_{m}=\left\lfloor b_{m}^{\frac{\mu}{3-s}}\right\rfloor$ so that Eq (5.8) becomes

$$
\Phi_{s, N_{m}}(\alpha)=\frac{1}{1-s} b^{\frac{(1-s) \mu}{3-s}-1}+\mathcal{O}\left(b_{m}\right)+\mathcal{O}(1) .
$$


The condition on $\mu$ ensures that $\frac{(1-s) \mu}{3-s}-1>1$ and thus

$$
\limsup _{N \rightarrow+\infty} \Phi_{s, N}(\alpha)=+\infty \text {. }
$$

In fact, (5.9) even implies that for any $\varepsilon>0$, we have

$$
\limsup _{N \rightarrow+\infty} \frac{\Phi_{s, N}(\alpha)}{N^{(1-s)-\frac{3-s}{\mu}-\varepsilon}}=+\infty .
$$

The reader will easily adapt this computation to the case of Liouville numbers where she will replace $\mu$ by a sequence $\left(\mu_{m}\right)_{m}$ that tends to $+\infty$ with $m$. In this case, we obtain

$$
\limsup _{N \rightarrow+\infty} \frac{\Phi_{s, N}(\alpha)}{N^{(1-s)-\varepsilon}}=+\infty \text {. }
$$

Again, this contruction can be made for a dense set of Liouville numbers.

5.5. Proof of $(v)$. To prove the divergence of $\Phi_{s, N}(\alpha)$ for any $\alpha$ and $s \leq 0$, it is enough to prove that that $\sum_{m=1}^{n} \cos (2 \pi m n \alpha)$ does not tend to 0 as $n \rightarrow+\infty$. For a rational $\alpha=a / b$, this is immediate because, for any integer $n$, we have

$$
\sum_{m=1}^{b n} \cos (2 \pi m n b \alpha)=\sum_{m=1}^{b n} \cos (2 \pi m n a)=b n,
$$

hence

$$
\limsup _{n \rightarrow+\infty} \frac{1}{n} \sum_{m=1}^{n} \cos \left(2 \pi m n \frac{a}{b}\right)=1
$$

For $\alpha$ irrational, this is a consequence of the following lemma.

Lemma 6. For any irrational number $\alpha$, we have

$$
\limsup _{n \rightarrow+\infty} \frac{1}{n} \sum_{m=1}^{n} \cos (2 \pi m n \alpha)=\frac{L(\alpha)}{2 \pi} \sin \left(\frac{2 \pi}{L(\alpha)}\right) \neq 0 .
$$

Proof. We have

$$
\begin{aligned}
\frac{1}{n} \sum_{m=1}^{n} \cos (2 \pi m n \alpha) & =\frac{\cos (\pi n(n+1) \alpha) \sin \left(\pi n^{2} \alpha\right)}{n \sin (\pi n \alpha)} \\
& =\frac{\cos (\pi n \alpha) \sin \left(2 \pi n^{2} \alpha\right)}{2 n \sin (\pi n \alpha)}-\frac{\sin \left(\pi n^{2} \alpha\right)^{2}}{n} \\
& =\frac{\cos (\pi\|n \alpha\|) \sin (2 \pi n\|n \alpha\|)}{2 n \sin (\pi\|n \alpha\|)}+\mathcal{O}\left(\frac{1}{n}\right) .
\end{aligned}
$$


The quantity $\frac{\cos (\pi\|n \alpha\|) \sin (2 \pi n\|n \alpha\|)}{2 n \sin (\pi\|n \alpha\|)}$ is maximal when $\|n \alpha\|$ is close to 0 and $n\|n \alpha\|$ not too close to 0 , and thus

$$
\begin{aligned}
\limsup _{n \rightarrow+\infty} \frac{1}{n} \sum_{m=1}^{n} \cos (2 \pi m n \alpha) & =\limsup _{n \rightarrow+\infty} \frac{\sin (2 \pi n\|n \alpha\|)}{2 n \sin (\pi\|n \alpha\|)} \\
& =\limsup _{n \rightarrow+\infty} \frac{\sin (2 \pi n\|n \alpha\|)}{2 \pi n\|n \alpha\|} \\
& =\frac{L(\alpha)}{2 \pi} \sin \left(\frac{2 \pi}{L(\alpha)}\right) .
\end{aligned}
$$

Moreover, since $L(\alpha) \geq \sqrt{5}$, we have $0<2 \pi / L(\alpha)<\pi$ and thus $\sin \left(\frac{2 \pi}{L(\alpha)}\right) \neq 0$.

\section{Proof of Theorem 2}

6.1. Proof of $(i)$. We fix a rational number $a / b$ with $\operatorname{gcd}(a, b)=1$ and $b=1$. Using the Fourier expansion of $\|\alpha\|$ as for $F_{N}(\alpha)$, we obtain

$$
\frac{1}{H_{N}(s)} G_{s, N}\left(\frac{a}{b}\right)=\frac{1}{4}-\frac{2}{\pi^{2}} \sum_{\ell=0}^{\infty} \frac{1}{(2 \ell+1)^{2}} \frac{1}{H_{N}(s)} \Phi_{s, N}\left((2 \ell+1) \frac{a}{b}\right) .
$$

We now use Eq. (5.1), which says that, provided $\operatorname{gcd}(u, v)=1$ and $v \geq 1$, then

$$
\Phi_{s, N}\left(\frac{u}{v}\right)=\frac{1}{v} H_{N}(s)+\mathcal{O}(v)
$$

where the constant depends only on $s$. Hence, if $u$ and $v$ are not coprime, we have

$$
\Phi_{s, N}\left(\frac{u}{v}\right)=\frac{\operatorname{gcd}(u, v)}{v} H_{N}(s)+\mathcal{O}\left(\frac{v}{\operatorname{gcd}(u, v)}\right)
$$

It follows that

$$
\frac{1}{H_{N}(s)} G_{s, N}\left(\frac{a}{b}\right)=\frac{1}{4}-\frac{2}{\pi^{2}} \sum_{\ell=0}^{\infty} \frac{\operatorname{gcd}(b, 2 \ell+1)}{b(2 \ell+1)^{2}}+\mathcal{O}\left(\frac{b}{H_{N}(s)}\right)
$$

where the constant depends only on $s$.

We now prove the alternative expression of the main term as a product. The principle is very similar to what was done earlier. We observe that the arithmetic function $\ell \mapsto \operatorname{gcd}(b, \ell)$ 
is multiplicative and thus that, for any complex number $s$ such that $\operatorname{Re}(s)>1$,

$$
\begin{aligned}
& \sum_{\ell=0}^{\infty} \frac{\operatorname{gcd}(b, 2 \ell+1)}{(2 \ell+1)^{s}}=\prod_{p \geq 3}\left(\sum_{k=0}^{\infty} \frac{\operatorname{gcd}\left(b, p^{k}\right)}{p^{k s}}\right) \\
& =\prod_{\substack{p \geq 3 \\
p \mid b}}\left(\sum_{k=0}^{v_{p}(b)} \frac{p^{k}}{p^{k s}}+\sum_{k=v_{p}(b)+1}^{\infty} \frac{p^{v_{p}(b)}}{p^{k s}}\right) \cdot \prod_{\substack{p \geq 3 \\
p \nmid b}}\left(\sum_{k=0}^{\infty} \frac{1}{p^{k s}}\right) \\
& =\zeta(s)\left(1-\frac{1}{2^{s}}\right) \prod_{\substack{p \geq 3 \\
p \mid b}}\left(\frac{1}{p^{(s-1) v_{p}(b)}}\left(\frac{p^{(s-1)\left(v_{p}(b)+1\right)}-1}{p^{s-1}-1}-\frac{1}{p^{s}-1}\right)\left(1-\frac{1}{p^{s}}\right)\right) .
\end{aligned}
$$

Using this for $s=2$, we find

$$
\lim _{N \rightarrow+\infty} \frac{1}{H_{N}(s)} G_{s, N}\left(\frac{a}{b}\right)=\frac{1}{4}-\frac{1}{4 b} \prod_{\substack{p \geq 3 \\ p \mid b}}\left(\frac{\left(p^{v_{p}(b)+1}-1\right)(p+1)-1}{p^{v_{p}(b)+2}}\right),
$$

which is a rational number $<\frac{1}{4}$.

6.2. Proof of $(i i)$. As seen at the beginning of the proof of $(i)$, we have

$$
\mathscr{G}_{s, N}(\alpha):=G_{s, N}(\alpha)-\frac{1}{4} H_{N}(s)=-\frac{2}{\pi^{2}} \sum_{\ell=0}^{\infty} \frac{\Phi_{s, N}((2 \ell+1) \alpha)}{(2 \ell+1)^{2}} .
$$

To justify that we can pass to the limit $N \rightarrow+\infty$ under the sum sign, we will use Tannery's theorem, which is a version of Lebesgue dominated convergence theorem for series: Let $\left(A_{n}(k)\right)_{n \geq 0}$ be a sequences of complex numbers that depends on an integer parameter $k \geq 0$. Let us assume that

- for all $n \geq 0, \lim _{k \rightarrow+\infty} A_{n}(k)$ exists and is finite;

- for all $n \geq 0$, there exists $M_{n}$ such that $\left|A_{n}(k)\right| \leq M_{n}$ for all $k \geq 0$ and such that $\sum_{n} M_{n}$ is convergent.

Then, $\lim _{k \rightarrow+\infty} \sum_{n=0}^{\infty} A_{n}(k)=\sum_{n=0}^{\infty} \lim _{k \rightarrow+\infty} A_{n}(k)<\infty$.

We will also need the following lemma.

Lemma 7. ( $i)$ For any $\alpha \in \mathscr{A}_{1}$ with a finite irrationality exponent $\mu(\alpha)$, any integer $N \geq 1$ and any integer $k \geq 1$, we have $\left|\Phi_{1, N}(k \alpha)\right| \ll \log (k+1)$, where the constant depends on $\alpha$.

(ii) Given $s \in(0,1)$, for any $\alpha \in \mathscr{A}_{s}$ with an irrationality exponent $\mu(\alpha)<1+\frac{1}{1-s}$, any integer $N \geq 1$ and any integer $k \geq 1$, we have $\left|\Phi_{s, N}(k \alpha)\right| \ll k^{(\mu(\alpha)-1)(1-s)}$, where the constant depends on $\alpha$ and $s$.

Proof. Using the inequality (5.2) and items (iii) and (iv) of Proposition 1, we find that

$$
\left|\Phi_{1, N}(\alpha)\right| \ll \mu(\alpha)+\log c(\alpha)
$$


and

$$
\left|\Phi_{s, N}(\alpha)\right| \ll \frac{c(\alpha)^{1-s}}{1-\sqrt{2}^{(\mu(\alpha)-1)(1-s)-1}},
$$

where the first constant is absolute and the second one depends on $s$. Furthermore, it is straitghforward to see that if $\alpha$ has a finite irrationality exponent, then for all integer $k \geq 1$, the irrational number $k \alpha$ also has a finite irrationality exponent and that we can take $\mu(k \alpha)$ and $c(k \alpha))$ such that

$$
\mu(k \alpha)=\mu(\alpha), \quad c(k \alpha) \leq c(\alpha) k^{\mu(\alpha)-1} .
$$

Hence,

$$
\begin{aligned}
& \left|\Phi_{1, N}(k \alpha)\right| \ll \mu(k \alpha)+\log c(k \alpha) \\
& \quad \leq \mu(\alpha)+(\mu(\alpha)-1) \log (k)+\log c(\alpha) \ll \log (k+1),
\end{aligned}
$$

and similarly

$$
\left|\Phi_{s, N}(k \alpha)\right| \ll \frac{c(k \alpha)^{1-s}}{1-\sqrt{2}^{(\mu(k \alpha)-1)(1-s)-1}} \leq \frac{c(\alpha)^{1-s} k^{(\mu(\alpha)-1)(1-s)}}{1-\sqrt{2}^{(\mu(\alpha)-1)(1-s)-1}} \ll k^{(\mu(\alpha)-1)(1-s)},
$$

where both constants depend on $\alpha$, and also $s$ for the second.

We can now easily finish the proof of item $(i i)$ of Theorem 2 . Indeed, in the case $s=1$, for any $\alpha \in \mathscr{A}_{1}$ with finite irrationality exponent, for any integer $\ell \geq 0,(2 \ell+1) \alpha$ also has a finite irrationality exponent and, therefore, belongs to $\mathscr{A}_{1}$ (by Lemma $1,(i)$ ). Hence $\Phi_{1, N}((2 \ell+1) \alpha)$ converges to $\Phi_{1}((2 \ell+1) \alpha)$ and by $(i)$ of Lemma 7 , we have

$$
\left|\frac{\Phi_{1, N}((2 \ell+1) \alpha)}{(2 \ell+1)^{2}}\right| \ll \frac{\log (\ell+1)}{(2 \ell+1)^{2}},
$$

where the right-hand side is the term of convergent series. By Tannery's theorem, we therefore have

$$
\begin{aligned}
\lim _{N \rightarrow+\infty} \mathscr{G}_{s, N}(\alpha) & =-\lim _{N \rightarrow+\infty} \frac{2}{\pi^{2}} \sum_{\ell=0}^{\infty} \frac{\Phi_{1, N}((2 \ell+1) \alpha)}{(2 \ell+1)^{2}} \\
& =-\frac{2}{\pi^{2}} \sum_{\ell=0}^{\infty} \frac{\Phi_{1}((2 \ell+1) \alpha)}{(2 \ell+1)^{2}} .
\end{aligned}
$$

In the case $s \in(0,1)$, for any $\alpha \in \mathscr{A}_{s}$ with an irrationality exponent $\mu(\alpha)<1+\frac{1}{1-s}$, for any integer $\ell \geq 0,(2 \ell+1) \alpha$ also has an irrationality exponent $\mu((2 \ell+1) \alpha)=\mu(\alpha)<1+\frac{1}{1-s}$ and, therefore, belongs to $\mathscr{A}_{s}$ (by Lemma 1, (ii)). Hence $\Phi_{s, N}((2 \ell+1) \alpha)$ converges to $\Phi_{s}((2 \ell+1) \alpha)$ and by $(i i)$ of Lemma 7 , we have

$$
\left|\frac{\Phi_{s, N}((2 \ell+1) \alpha)}{(2 \ell+1)^{2}}\right| \ll \frac{1}{\ell^{2-(\mu(\alpha)-1)(1-s)}},
$$


where the right-hand side is the term of convergent series because $1>(\mu(\alpha)-1)(1-s)$. Again, by Tannery's theorem, we therefore have

$$
\begin{aligned}
\lim _{N \rightarrow+\infty} \mathscr{G}_{s, N}(\alpha) & =-\lim _{N \rightarrow+\infty} \frac{2}{\pi^{2}} \sum_{\ell=0}^{\infty} \frac{\Phi_{s, N}((2 \ell+1) \alpha)}{(2 \ell+1)^{2}} \\
& =-\frac{2}{\pi^{2}} \sum_{\ell=0}^{\infty} \frac{\Phi_{s}((2 \ell+1) \alpha)}{(2 \ell+1)^{2}} .
\end{aligned}
$$

6.3. Proof of $(i i i)$. The almost sure convergence of $\mathscr{G}_{s, N}$ to $\mathscr{G}_{s}$ is a consequence of $(i i)$ because the sets $\mathscr{A}_{s}$ all have measure 1. It remains to prove the convergence in $L^{2}(0,1)$. Firstly, we note that for any integer $k \geq 0$ and any integers $M \geq N \geq 1$, we have $\left\|\Phi_{s, M}((2 k+1) \alpha)-\Phi_{s, N}((2 k+1) \alpha)\right\|_{2}=\left\|\Phi_{s, M}(\alpha)-\Phi_{s, N}(\alpha)\right\|_{2}$ by the 1-periodicity of the $\Phi_{s, N}$. Therefore,

$$
\begin{aligned}
\left\|\mathscr{G}_{s, M}(\alpha)-\mathscr{G}_{s, N}(\alpha)\right\|_{2} & \leq \frac{2}{\pi^{2}} \sum_{k=0}^{\infty} \frac{1}{(2 k+1)^{2}}\left\|\Phi_{s, M}((2 k+1) \alpha)-\Phi_{s, N}((2 k+1) \alpha)\right\|_{2} \\
& \leq \frac{2}{\pi^{2}} \sum_{k=0}^{\infty} \frac{1}{(2 k+1)^{2}}\left\|\Phi_{s, M}(\alpha)-\Phi_{s, N}(\alpha)\right\|_{2} \\
& \leq \frac{1}{4}\left\|\Phi_{s, M}(\alpha)-\Phi_{s, N}(\alpha)\right\|_{2} .
\end{aligned}
$$

Since the right hand side converges to 0 by Theorem 1 when $M \geq N \rightarrow+\infty$, we have obtained the convergence of the $\mathscr{G}_{s, N}$ in $L^{2}(0,1)$ to a function that can be nothing but $\mathscr{G}_{s}$.

6.4. Proof of $(i v)$. We will use the same method as in the proof of item $(i v)$ in Theorem 1. The proof is based on the "identity" (6.2) given in Lemma 8 below. We will then leave most of the details to the reader as no new idea will be involved.

Lemma 8. Let us fix $s \in(0,1]$, an irrational number $\alpha$ and a rational number $a / b$ with $\operatorname{gcd}(a, b)=1$ and $b \geq 1$. For any integers $M \geq 1, N \geq 1$ we have

$$
\begin{aligned}
G_{s, N}(\alpha)=\left(\frac{1}{4}-\frac{2}{\pi^{2}} \sum_{\ell=0}^{\infty} \frac{\operatorname{gcd}(b, 2 \ell+1)}{b(2 \ell+1)^{2}}\right) H_{N}(s) & \\
& +\mathcal{O}(b)+\mathcal{O}\left(\frac{H_{N}(s)}{M}\right)+\mathcal{O}\left(H_{M} N^{3-s}\left|\alpha-\frac{a}{b}\right|\right),
\end{aligned}
$$

where the implicit constants depend at most on $s$.

Proof. Nota Bene: As the reader will check, all the implicit constants below depend at most on $s$. 
Firstly, starting from the definition, we find that, for any integer $M \geq 1$,

$$
\begin{aligned}
& \left|G_{s, N}(\alpha)-G_{s, N}\left(\frac{a}{b}\right)\right| \\
& \qquad \leq \frac{2}{\pi^{2}}\left(\sum_{\ell=0}^{M}+\sum_{\ell=M+1}^{\infty}\right) \frac{1}{(2 \ell+1)^{2}}\left|\Phi_{s, N}((2 \ell+1) \alpha)-\Phi_{s, N}\left((2 \ell+1) \frac{a}{b}\right)\right| .
\end{aligned}
$$

Secondly, using the upper bound (5.3), we have

$$
\begin{aligned}
\sum_{\ell=0}^{M} \frac{1}{(2 \ell+1)^{2}}\left|\Phi_{s, N}((2 \ell+1) \alpha)-\Phi_{s, N}\left((2 \ell+1) \frac{a}{b}\right)\right| & \ll N^{3-s}\left|\alpha-\frac{a}{b}\right| \sum_{\ell=0}^{M} \frac{1}{2 \ell+1} \\
& \ll H_{M} N^{3-s}\left|\alpha-\frac{a}{b}\right| .
\end{aligned}
$$

Thirdly, we know that $\left|\Phi_{s, N}(\alpha)\right| \leq H_{N}(s)$ in all situations. Hence,

$$
\begin{aligned}
\sum_{\ell=M+1}^{\infty} \frac{1}{(2 \ell+1)^{2}} \mid \Phi_{s, N}((2 \ell+1) \alpha)-\Phi_{s, N} & \left((2 \ell+1) \frac{a}{b}\right) \mid \\
& \ll H_{N}(s) \sum_{\ell=M+1}^{\infty} \frac{1}{(2 \ell+1)^{2}} \ll \frac{H_{N}(s)}{M} .
\end{aligned}
$$

Therefore, we have obtained

$$
G_{s, N}(\alpha)=G_{s, N}\left(\frac{a}{b}\right)+\mathcal{O}\left(\frac{H_{N}(s)}{M}\right)+\mathcal{O}\left(H_{M} N^{3-s}\left|\alpha-\frac{a}{b}\right|\right)
$$

and the lemma follows using (6.1).

To conclude the proof of item $(i v)$, we first choose a sequence of rational $\left(a_{m} / b_{m}\right)_{m}$ that converges to $\alpha$, with $\left(a_{m}, b_{m}\right)=1, b_{m} \geq 1$. Then, we take $M=\left\lfloor H_{N}(s)\right\rfloor$, so that after some simplifications, (6.2) becomes

$$
G_{s, N}(\alpha)=\left(\frac{1}{4}-\frac{2}{\pi^{2}} \sum_{\ell=0}^{\infty} \frac{\operatorname{gcd}\left(b_{m}, 2 \ell+1\right)}{b_{m}(2 \ell+1)^{2}}\right) H_{N}(s)+\mathcal{O}\left(b_{m}\right)+\mathcal{O}\left(\log (N) N^{3-s}\left|\alpha-\frac{a_{m}}{b_{m}}\right|\right) .
$$

Since

it follows that

$$
\frac{2}{\pi^{2} b_{m}} \leq \frac{2}{\pi^{2}} \sum_{\ell=0}^{\infty} \frac{\operatorname{gcd}\left(b_{m}, 2 \ell+1\right)}{b_{m}(2 \ell+1)^{2}}
$$

$$
G_{s, N}(\alpha)-\frac{1}{4} H_{N}(s) \leq-\frac{2 H_{N}(s)}{\pi^{2} b_{m}}+\mathcal{O}\left(b_{m}\right)+\mathcal{O}\left(\log (N) N^{3-s}\left|\alpha-\frac{a_{m}}{b_{m}}\right|\right) .
$$

For $s=1$ or $s \in(0,1)$, we now take for $\alpha$ the same reals as those used during the proof of item $(i v)$ of Theorem 1 and with the same choices for $N$ in function of $b_{m}$. In (6.3), the main term is $-\frac{2 H_{N}(s)}{\pi^{2} b_{m}}$ and we obtain the divergence at the rate indicated for irrational 
numbers with irrationality exponent $m(\alpha)>2+\frac{4}{1-s}$ when $s \in(0,1)$, or for a dense set of Liouville numbers when $s=1$.

6.5. Proof of $(i v)$. Since $L(\alpha) \geq \sqrt{5}$ for any irrational number $\alpha$, it follows from item (iv) of Theorem 4 that

$$
\liminf _{n \rightarrow+\infty} F_{n}(\alpha)<\frac{1}{4}
$$

Thus, for any $s \leq 0$, the sequence $n^{-s}\left(F_{n}(\alpha)-\frac{1}{4}\right)$ does not tend to zero and a fortiori the series $\sum_{n} n^{-s}\left(F_{n}(\alpha)-\frac{1}{4}\right)$ does not converge.

The divergence for $\alpha$ a rational number is a consequence of the oscillating (and nearly periodic) behavior of $F_{n}(\alpha)-\frac{1}{4}$ as shown in item (ii) of Theorem 4 .

\section{Proof of Theorem 3}

7.1. Proof of $(i)$. The Fourier expansion of $\Phi_{s, N}(\alpha)$ is

$$
\begin{aligned}
\Phi_{s, N}(\alpha) & =\sum_{k=1}^{N^{2}} \cos (2 \pi k \alpha) \sum_{\substack{1 \leq m \leq n \leq N \\
m n=k}} \frac{1}{n^{s+1}}=\sum_{k=1}^{N^{2}} \cos (2 \pi k \alpha) \sum_{\substack{n \mid k \\
\sqrt{k} \leq n \leq N}} \frac{1}{n^{s+1}} \\
& =\sum_{k=1}^{\infty} \cos (2 \pi k \alpha) \sum_{\substack{n \mid k \\
\sqrt{k} \leq n \leq N}} \frac{1}{n^{s+1}}
\end{aligned}
$$

and the difficulty is to justify that we can pass to the limit under the sum sign.

By Carleson's theorem [5], we know that the Fourier expansion of $\Phi_{s}$ converges almost surely to $\Phi_{s}$ because $\Phi_{s} \in L^{2}(0,1)$. We will give a direct proof of the almost sure convergence that avoids this difficult theorem.

Set

$$
\widehat{S}_{s, N}(\alpha)=\sum_{k=1}^{N} \widehat{\phi}_{s, k} \cos (2 \pi k \alpha)
$$

where $\widehat{\phi}_{s, k}:=\sum_{n \mid k, n \geq \sqrt{k}} \frac{1}{n^{s+1}}$. We will show that, for all $\alpha \in \mathscr{B}_{s}$,

$$
\lim _{N \rightarrow+\infty} \widehat{S}_{s, N}(\alpha)=\Phi_{s}(\alpha) .
$$

This will prove that the trigonometric series

$$
\widehat{S}_{s}(\alpha):=\sum_{k=1}^{\infty} \widehat{\phi}_{s, k} \cos (2 \pi k \alpha)
$$

converges almost surely to $\Phi_{s}(\alpha)$. It will remain to show that $\widehat{S}_{s}$ is the Fourier series of $\Phi_{s}$, i.e. that the coefficients $\widehat{\phi}_{k, s}$ coincide the Fourier coefficients $\phi_{k, s}$ of $\Phi_{s}$ computed by the usual integrals. 
We now pass to the details

- Almost sure convergence of a trigonometric series to $\Phi_{s}$.

For any real number $\alpha$, we observe that

$$
\begin{aligned}
\widehat{S}_{s, N}(\alpha) & =\sum_{n=1}^{N} \frac{1}{n^{s+1}} \sum_{1 \leq k \leq n^{2}, k \leq N, n \mid k} \cos (2 \pi k \alpha)=\sum_{n=1}^{N} \frac{1}{n^{s+1}} \sum_{1 \leq k \leq n, k \leq N / n} \cos (2 \pi k n \alpha) \\
& =\sum_{1 \leq n \leq \sqrt{N}} \frac{1}{n^{s+1}} \sum_{k=1}^{n} \cos (2 \pi k n \alpha)+\sum_{\sqrt{N<n \leq N}} \frac{1}{n^{s+1}} \sum_{1 \leq k \leq N / n} \cos (2 \pi k n \alpha) \\
& =\sum_{1 \leq n \leq \sqrt{N}} \frac{1}{n^{s+1}} \sum_{k=1}^{n} \cos (2 \pi k n \alpha)+\mathcal{O}\left(\sum_{\sqrt{N<n \leq N}} \frac{1}{n^{s+1}\|n \alpha\|}\right) .
\end{aligned}
$$

In the last step, we have used the following fact: we have

$$
\sum_{1 \leq k \leq N / n} \cos (2 \pi k n \alpha)=\mathcal{O}\left(\frac{1}{|\sin (\pi n \alpha)|}\right)=\mathcal{O}\left(\frac{1}{\|n \alpha\|}\right),
$$

where the constants are absolute, so that

$$
\sum_{\sqrt{N}<n \leq N} \frac{1}{n^{s+1}} \sum_{1 \leq k \leq N / n} \cos (2 \pi k n \alpha)=\mathcal{O}\left(\sum_{\sqrt{N}<n \leq N} \frac{1}{n^{s+1}|| n \alpha||}\right) .
$$

We have thus obtained the equality

$$
\widehat{S}_{s, N}(\alpha)=\Phi_{\lfloor\sqrt{N}\rfloor, s}(\alpha)+\mathcal{O}\left(\sum_{\sqrt{N}<n \leq N} \frac{1}{n^{s+1}|| n \alpha||}\right) .
$$

If $\alpha \in \mathscr{A}_{s}$, then $\Phi_{\lfloor\sqrt{N}\rfloor, s}(\alpha)$ converges to $\Phi_{s}(\alpha)$ while if $\alpha \in \mathscr{B}_{s}$, then the series $\sum_{n} \frac{1}{n^{s+1}\|n \alpha\|}$ is convergent by Kruse's inequality (4.4). Therefore, $\lim _{N} \widehat{S}_{s, N}(\alpha)$ exists and is equal to $\Phi_{s}(\alpha)$ for all irrational numbers $\alpha \in \mathscr{A}_{s} \cap \mathscr{B}_{s}=\mathscr{B}_{s}$ (at least).

- Convergence of $\widehat{S}_{s}$ to $\Phi_{s}$ in $L^{2}(0,1)$. It is clear that, for any $s \in(0,1]$,

$$
\left|\phi_{s, k}\right| \leq \frac{1}{k^{(s+1) / 2}} \sum_{n \mid k} 1 \ll \frac{e^{c \log (k) / \log \log (k)}}{k^{(s+1) / 2}} .
$$

Hence $\sum_{k} \phi_{k, s}^{2}<\infty$ and by the Fischer-Riesz's Theorem, the series $\widehat{S}_{s}$ converges in $L^{2}(0,1)$. Its $L^{2}$-sum is $\Phi_{s}$ because it converges to it also almost surely.

- Fourier coefficients of $\Phi_{s}$. Let $\left(c_{s, k}\right)_{k \in \mathbb{Z}}$ and $\left(\widehat{c}_{s, k}\right)_{k \in \mathbb{Z}}$ denote the Fourier coefficients of $\Phi_{s}$ and $\widehat{S}_{s}$ respectively (which are already known to be 0 for $k=0$ and $k$ odd). Since both functions belong to $L^{2}(0,1)$, the coefficients can be computed by the usual integrals and by the Cauchy-Schwarz inequality, we have for any $k \in \mathbb{Z},\left|c_{s, k}-\widehat{c}_{k, s}\right| \leq\left\|\Phi_{s}-\widehat{S}_{s}\right\|_{2}=0$. Hence, $\widehat{S}_{s}$ is the Fourier series of $\Phi_{s}$. 
7.2. Proof of $(i i)$. We now know that $\widehat{S}_{s, N}(\alpha)=S_{s, N}(\alpha)$ and we start again with the identity (7.1):

$$
S_{s, N}(\alpha)=\sum_{1 \leq n \leq \sqrt{N}} \frac{1}{n^{s+1}} \sum_{k=1}^{n} \cos (2 \pi k n \alpha)+\sum_{\sqrt{N}<n \leq N} \frac{1}{n^{s+1}} \sum_{1 \leq k \leq N / n} \cos (2 \pi k n \alpha) .
$$

We now specify $\alpha=a / b \in \mathbb{Q}$ with $\operatorname{gcd}(a, b)=1, b \geq 1$.

We use again a computation done during the proof of Theorem 4, (ii):

$$
\sum_{1 \leq k \leq N / n} \cos \left(2 \pi k n \frac{a}{b}\right)=\frac{N}{b n} \sum_{j=1}^{b} \cos \left(2 \pi j n \frac{a}{b}\right)+\mathcal{O}(b) .
$$

Hence, using the fact that $\sum_{j=1}^{b} \cos \left(2 \pi j n \frac{a}{b}\right)=0$, resp. $=b$, if $b \nmid n$, resp. $b \mid n$, we obtain that

$$
\begin{aligned}
S_{s, N}\left(\frac{a}{b}\right) & =\Phi_{\lfloor\sqrt{N}\rfloor, s}\left(\frac{a}{b}\right)+N \sum_{\substack{\sqrt{N}<n \leq N \\
b \mid n}} \frac{1}{n^{s+2}}+\mathcal{O}\left(b \sum_{\sqrt{N}<n \leq N} \frac{1}{n^{s+1}}\right) \\
& =\frac{1}{b} H_{\lfloor\sqrt{N}\rfloor}(s)(1+o(1))+\frac{1}{b(s+1)} N^{\frac{1-s}{2}}(1+o(1))+\mathcal{O}(b) .
\end{aligned}
$$

(In (7.2), the implicit constant is absolute.)

If $s \in(0,1)$, then since $H_{\lfloor\sqrt{N\rfloor}}(s) \sim \frac{1}{1-s} N^{\frac{1-s}{2}}$, it follows that

$$
\lim _{N \rightarrow+\infty} \frac{1}{H_{\lfloor\sqrt{N}\rfloor}(s)} S_{s, N}\left(\frac{a}{b}\right)=\frac{2}{b(1+s)} .
$$

If $s=1$, then the term $\frac{1}{b(s+1)} N^{\frac{1-s}{2}}(1+o(1))$ is simply $\mathcal{O}(1)$, hence

$$
\lim _{N \rightarrow+\infty} \frac{1}{H_{\lfloor\sqrt{N}\rfloor}} S_{1, N}\left(\frac{a}{b}\right)=\frac{1}{b}
$$

and (7.3) also holds for $s=1$. This completes the proof of $(i i)$.

7.3. Proof of $($ iii $)$. Item (iii) is proved by the same method as the one used to show that the sequence $\Phi_{1, N}$ does not converge for certain Liouville numbers: for this we use the approximations (5.1) and (7.2).

\section{GRaphS AND NUMERICAL VALUES}

In this section, we present approximate values of the function $\Phi_{1}(\alpha)$ for various values of $\alpha$, obtained using the freeware GP-Pari [10]. For this, we need to find an upper bound for the speed of convergence of the sequence $\Phi_{s, N}(\alpha)$ to its limit. We explain later why, despite an explicit bound for the speed of convergence of $\mathscr{G}_{s, N}(\alpha)$, we cannot compute values of $\mathscr{G}_{s}(\alpha)$. Some graphs are also displayed, obtained using the computer algebra freeware XCAS [22]. 


\subsection{Speed of convergence.}

Proposition 2. (i) If $\alpha \in \mathscr{A}_{1}$ has a finite irrationality exponent $\mu(\alpha)$, we have

$$
\begin{aligned}
& \left|\Phi_{1}(\alpha)-\Phi_{1, N}(\alpha)\right| \quad \leq \pi(1+\zeta(2))\left(3(1+\log c(\alpha)) \frac{\log \left(q_{m}\right)}{q_{m}}+5(\mu(\alpha)-1) \frac{\log \left(q_{m}\right)^{2}}{q_{m}}\right)
\end{aligned}
$$

for any integer $N$ such that $N \geq q_{m}$ with $m \geq 6$.

(ii) If $\alpha \in \mathscr{A}_{s}$ (for some $s \in(0,1)$ ) and has an irrationality exponent $\mu(\alpha)<1+\frac{1}{1-s}$, then

$$
\begin{aligned}
& \left|\Phi_{s}(\alpha)-\Phi_{s, N}(\alpha)\right| \\
& \quad \leq \frac{\pi(1+\zeta(s+1))}{(1-s) q_{m}^{1-(\mu(\alpha)-1)(1-s)}}\left(3\left(1+c(\alpha)^{1-s}\right) \log \left(q_{m}\right)+\frac{c(\alpha)^{1-s}}{1-\sqrt{2}^{(\mu(\alpha)-1)(1-s)-1}}\right) .
\end{aligned}
$$

for any integer $N$ such that $N \geq q_{m}$ with $m \geq 6$.

Proof. During the proof of item (ii) of Theorem 1, we saw that

$$
\left|\frac{\cos (\pi n(n+1) \alpha) \sin \left(\pi n^{2} \alpha\right)}{\sin (\pi n \alpha)}\right| \leq \frac{\pi}{2} \frac{\left\|n^{2} \alpha\right\|}{\|n \alpha\|}
$$

for any $n \geq 1$. Hence,

$$
\left|\Phi_{s}(\alpha)-\Phi_{s, N}(\alpha)\right| \leq \sum_{n=N}^{\infty}\left|\frac{\cos (\pi n(n+1) \alpha) \sin \left(\pi n^{2} \alpha\right)}{n^{s+1} \sin (\pi n \alpha)}\right| \leq \frac{\pi}{2} \sum_{n=N}^{\infty} \frac{\left\|n^{2} \alpha\right\|}{n^{s+1}\|n \alpha\|}
$$

and the conclusion follows by Proposition 1, (iii) and (iv).

The bounds given by Proposition 2 are good enough to provide a few digits of $\Phi_{s}(\alpha)$ with a computer for any given $\alpha$ and $s$ and with $N$ of a reasonable size. The situation is somewhat different for the computation of $\mathscr{G}_{s}(\alpha)$. It is possible to obtain explicit bounds for the difference $\left|\mathscr{G}_{s}(\alpha)-\mathscr{G}_{s, N}(\alpha)\right|$, using the fact that

$$
\left|\mathscr{G}_{s}(\alpha)-\mathscr{G}_{s, N}(\alpha)\right| \leq \frac{2}{\pi^{2}} \sum_{k=0}^{\infty} \frac{1}{(2 k+1)^{2}}\left|\Phi_{s}((2 k+1) \alpha)-\Phi_{s, N}((2 k+1) \alpha)\right| .
$$

We now use Proposition 2 to bound

$$
\Phi_{s}((2 k+1) \alpha)-\Phi_{s, N}((2 k+1) \alpha)
$$

but we have to be careful that the bound depends on $q_{m}=q_{m}((2 k+1) \alpha)$. There does not seem to exist general results providing a simple link between the sequences $\left(q_{m}(\alpha)\right)_{m}$ and $\left(q_{m}(\ell \alpha)\right)_{m}$, where $\ell$ is any positive integer. Therefore, we uniformize the bounds of Proposition 2 by means of the (already multiply used) inequality $q_{m}(\alpha) \geq 2^{(m-1) / 2}$. We don't write the exact result which seems useless in practice because, for example, to compute the first digit of $\mathscr{G}_{1}(\sqrt{2})$ one needs to compute $\mathscr{G}_{1,259717522849}(\sqrt{2})$. 
8.2. Explicit irrationality exponents. Our remaining problem is to find numerical expressions of $\mu(\alpha)$ and $c(\alpha)$ for a given $\alpha$. Unfortunately, there is no general recipe. In the proposition below, we collect a few results of the litterature that provide explicit values of these numbers for interesting irrational numbers. We emphasize that the values of $\mu(\alpha)$ below are not necessarily the best known (see [17] for more recent results) but, usually, the authors of these refinements take $c(\alpha)=1$ and state their results for large enough $q$, which is not the kind of result we need. It also happens in the case of algebraic numbers that bounds for $\mu(\alpha)$ close to Roth's result (which asserts that for any $\varepsilon>0, m(\alpha) \leq 2+\varepsilon$ but without an explicit expression of $c(\alpha, \varepsilon))$ have been obtained, but at the cost of increased values of $c(\alpha)$, which is not necessarily a good thing for numerical computations.

Proposition 3. (i) (Liouville's inequality [17]) Assume that $\alpha$ is a real algebraic irrational number of degree $d$, with minimal polynomial $P(X)=\sum_{j=0}^{d} c_{j} X^{j} \in \mathbb{Z}[X]$. For any rational number $p / q, q \geq 1$, wehave

$$
\left|\alpha-\frac{p}{q}\right| \geq \frac{1}{(|\alpha|+1)^{d-1} \sum_{j=1}^{d} j\left|c_{j}\right|} \cdot \frac{1}{q^{d}} .
$$

In other words, we can take $\mu(\alpha)=d$ and $c(\alpha)=(|\alpha|+1)^{d-1} \sum_{j=1}^{d} j\left|a_{j}\right|$.

(ii) (Baker's inequality [1]) For any rational number $p / q$ with $q \geq 1$, we have

$$
\left|\sqrt[3]{2}-\frac{p}{q}\right| \geq \frac{10^{-6}}{q^{2.955}}
$$

Hence we can take $\mu(\sqrt[3]{2})=2.955$ and $c(\sqrt[3]{2})=10^{6}$.

(iii) (Mignotte's inequalities [15]) For any rational number $p / q$ with $q \geq 2$, we have

$$
\left|\pi-\frac{p}{q}\right| \geq \frac{1}{q^{21}} \quad \text { and } \quad\left|\pi^{2}-\frac{p}{q}\right| \geq \frac{1}{q^{18}} .
$$

Hence we can take $\mu(\pi)=21, \mu\left(\pi^{2}\right)=18$ and $c(\pi)=c\left(\pi^{2}\right)=1$.

(iv) (Bundschuh's inequality [4]) For any rational number $p / q$ with $q \geq 1$, we have

$$
\left|e-\frac{p}{q}\right| \geq \frac{\log \log (4 q)}{18 q^{2} \log (4 q)} .
$$

Hence we can take $\mu(e)=2.1$ and $c(e)=77$.

Proof. For $(i i)$ to $(i v)$, we refer to the cited references. Item $(i)$ is also classical (in this or another form) but its proof is so short that we provide it here.

Let us assume that $\left|\alpha-\frac{p}{q}\right| \leq 1$. By the mean value theorem, there exists $\xi$ between $\alpha$ and $p / q$ such that

$$
P\left(\frac{p}{q}\right)=P(\alpha)-P\left(\frac{p}{q}\right)=P^{\prime}(\xi)\left(\alpha-\frac{p}{q}\right)
$$


Since the degree of $\alpha$ is at least 2 and $P$ is the minimal polynomial of $\alpha$, neither $P\left(\frac{p}{q}\right)$ nor $P^{\prime}(\xi)$ can be 0 . Hence, $\left|P\left(\frac{p}{q}\right)\right| \geq \frac{1}{q^{d}}$ and

$$
0<\left|P^{\prime}(\xi)\right| \leq \max (1,|\xi|)^{d-1} \sum_{j=1}^{d} j\left|c_{j}\right| .
$$

Therefore, we have

$$
\left|\alpha-\frac{p}{q}\right| \geq \frac{1}{\max (1,|\xi|)^{d-1} \sum_{j=1}^{d} j\left|c_{j}\right|} \cdot \frac{1}{q^{d}} .
$$

It remains to bound $\xi$. Since $\left|\alpha-\frac{p}{q}\right|<1$ and $\xi$ is between $\alpha$ and $\frac{p}{q}$, it is clear that $|\xi| \leq|\alpha|+1$. Hence $\max (1,|\xi|) \leq \max (1,1+|\alpha|)=1+|\alpha|$ and therefore

$$
\left|\alpha-\frac{p}{q}\right| \geq \frac{1}{(|\alpha|+1)^{d-1} \sum_{j=1}^{d} j\left|c_{j}\right|} \cdot \frac{1}{q^{d}} .
$$

Since this bound also holds when $\left|\alpha-\frac{p}{q}\right| \geq 1$, the result follows.

8.3. Graphs and numerical values of $\Phi_{s}(\alpha)$. In this section, we present approximations of various values of $\Phi_{s}(\alpha)$ for $s=1$. For this, we computed $\Phi_{s, N}(\alpha)$ with GP-Pari with $N$ and the reader will check using Propositions 2 and 3 that we get three, four or five significant digits as indicated.

We also present graphical representations of $\Phi_{s, 50000}$ for $s=1, \frac{1}{2}, \frac{1}{10}$. Since $\Phi_{s, N}$ are continous while $\Phi_{s}$ is (probably) not, these representations should be taken cautiously. However, they present some interesting features, coroborated by the results proved in the previous sections or the table below: we see that $\Phi_{s, N}(\alpha)$ converges for less values of $\alpha$ when $s$ is getting closer to 0$)$ and also that the absolute minimun of $\Phi_{s}$ seems to achieved at $\frac{\sqrt{5}-1}{2}$ and $\frac{3-\sqrt{5}}{2}$ in the four cases considered.

\begin{tabular}{|c|c|c|}
\hline$\alpha$ & $\Phi_{1}(\alpha)$ & $N$ \\
\hline$\frac{\sqrt{5}-1}{{ }^{2}}$ & -1.11153 & $2.05 \times 10^{9}$ \\
\hline$\sqrt{2}-1$ & -1.08588 & $2.08 \times 10^{9}$ \\
\hline$\frac{\sqrt{7565}-53}{82}$ & -1.08589 & $2.27 \times 10^{9}$ \\
\hline$\sqrt[3]{2}$ & $-0.1419(0)$ & $3.39 \times 10^{8}$ \\
\hline$e$ & $-0.3666(3)$ & $1.92 \times 10^{8}$ \\
\hline$\pi$ & $0.357(10)$ & $3.20 \times 10^{8}$ \\
\hline$\pi^{2}$ & $0.370(67)$ & $2.67 \times 10^{8}$ \\
\hline
\end{tabular}

The figures between parentheses are stable for a long time before reaching the indicated values of $N$, but our bound does not prove that they are correct. For the same reason, we do not mention our numerical results for $\Phi_{1 / 2}(\alpha)$. 


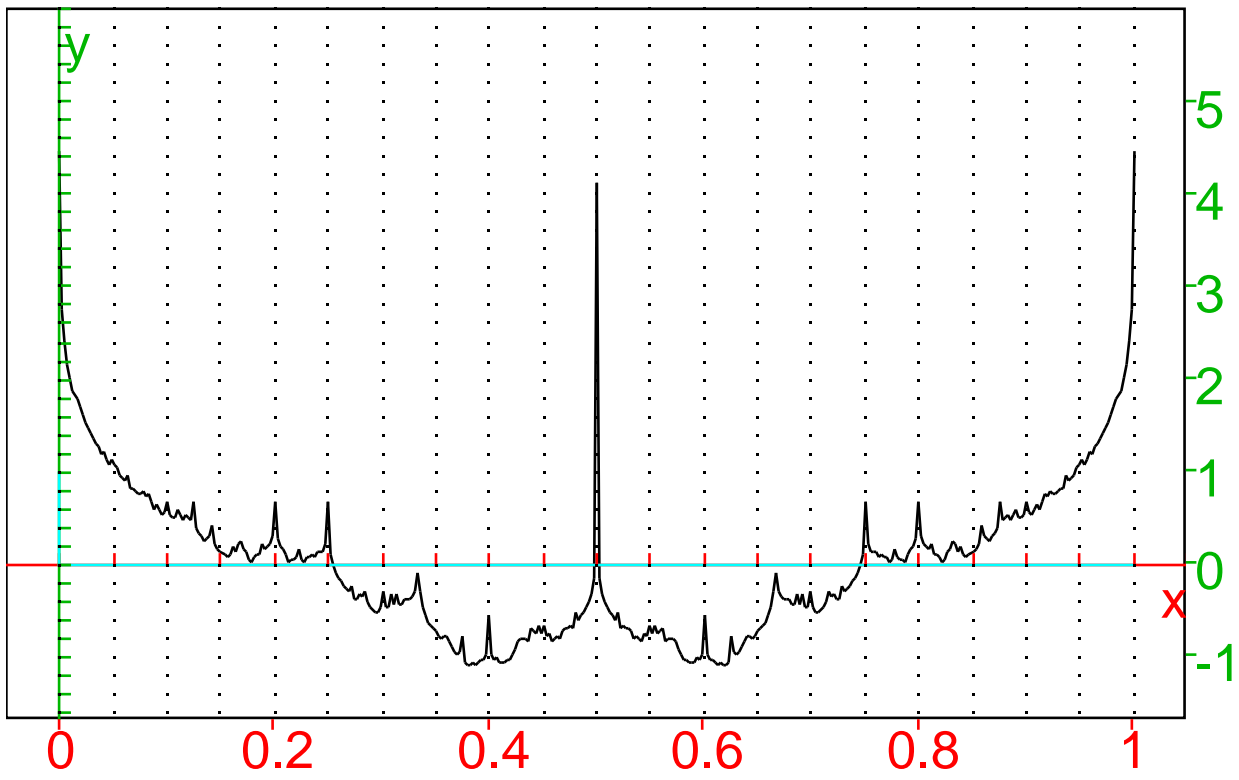

FiguRE 4. $\Phi_{1,50000}$

The choice of the quadratic number $\frac{\sqrt{7565}-53}{82}$ is not arbitrary. Indeed, in the Lagrange spectrum, it is the number with the fifth smallest Lagrange constant: we have

$$
L\left(\frac{\sqrt{5}-1}{2}\right)<L(\sqrt{2}-1)<L\left(\frac{\sqrt{221}-9}{14}\right)<L\left(\frac{\sqrt{1517}-23}{38}\right)<L\left(\frac{\sqrt{7565}-53}{82}\right)
$$

(see $\left[7\right.$, p. 10]). It is interesting to see that this order is not respected by $\Phi_{1}$ (and also by $\Phi_{1 / 2}$ if we believe our "unpublished" data):

$$
\Phi_{1}\left(\frac{\sqrt{5}-1}{2}\right)<\Phi_{1}\left(\frac{\sqrt{7565}-53}{82}\right)<\Phi_{1}(\sqrt{2}-1) .
$$

The function $\Phi_{s}$ does not have a clear diophantine signification, since it is just a brick used to define $\mathscr{G}_{s}$. But the fact that $\Phi_{1}\left(\frac{\sqrt{5}-1}{2}\right)$ seems to be a minimum value of $\Phi_{1}$ is puzzling.

8.4. Graphs and numerical values of $\mathscr{G}_{s}(\alpha)$. We present approximations to four digits for a few values of $\mathscr{G}_{1}(\alpha)$; they are computed from $\mathscr{G}_{1,30000}(\alpha)$. However, we cannot garantee that even a single digit after the decimal point is correct, even though our computations in Pari-GP suggest this is the case. It seems that

$$
\mathscr{G}_{1}\left(\frac{\sqrt{5}-1}{2}\right)=0.2169 \ldots, \mathscr{G}_{1}(\sqrt{2}-1)=0.2103 \ldots, \mathscr{G}_{1}\left(\frac{\sqrt{7565}-53}{82}\right)=0.2105 \ldots
$$

and again we observe that the order of Lagrange spectrum does not seem to be respected.

We also present graphical representations of $\mathscr{G}_{s, 200}$ for the same values of $1, \frac{1}{2}, \frac{1}{10}$. (For $s=1$, the plot is displayed in the Introduction.) The same precautions should be taken as for $\Phi_{s, 50000}$ concerning their interpretation. However, they present the same interesting 


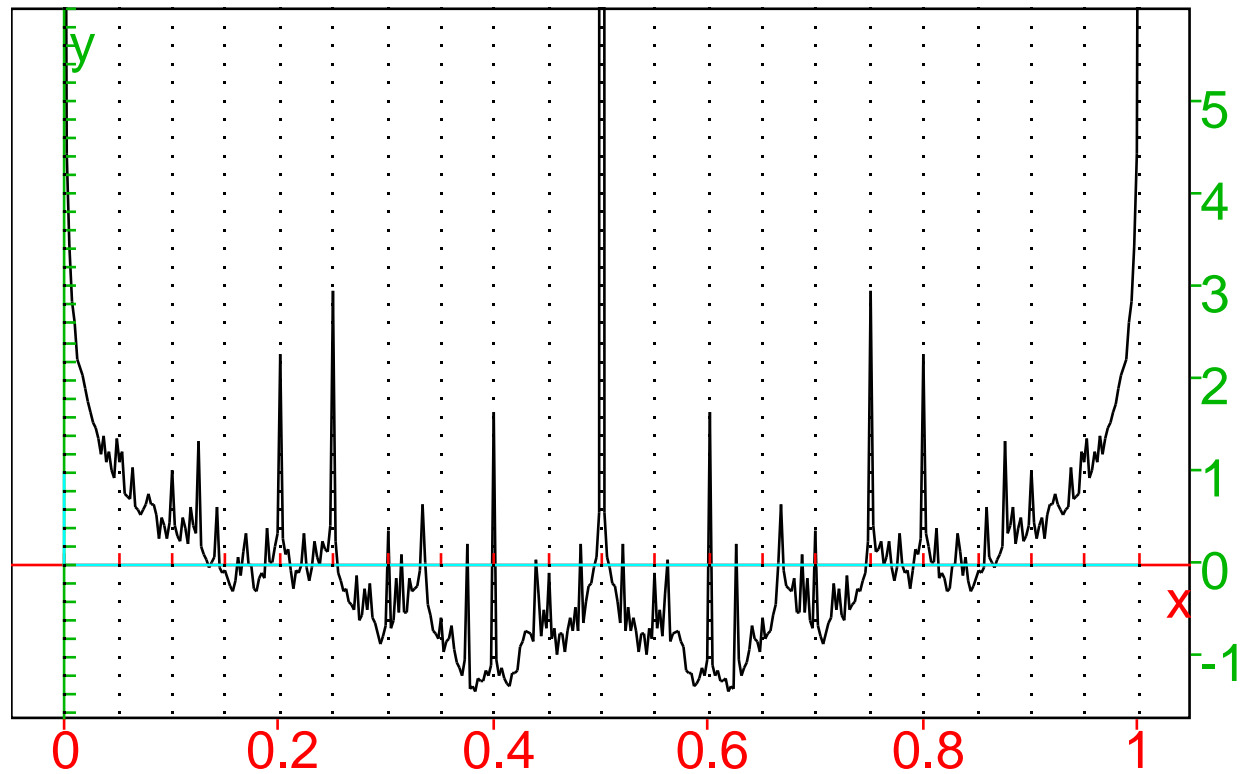

FIGURE 5. $\Phi_{\frac{1}{2}, 50000}$

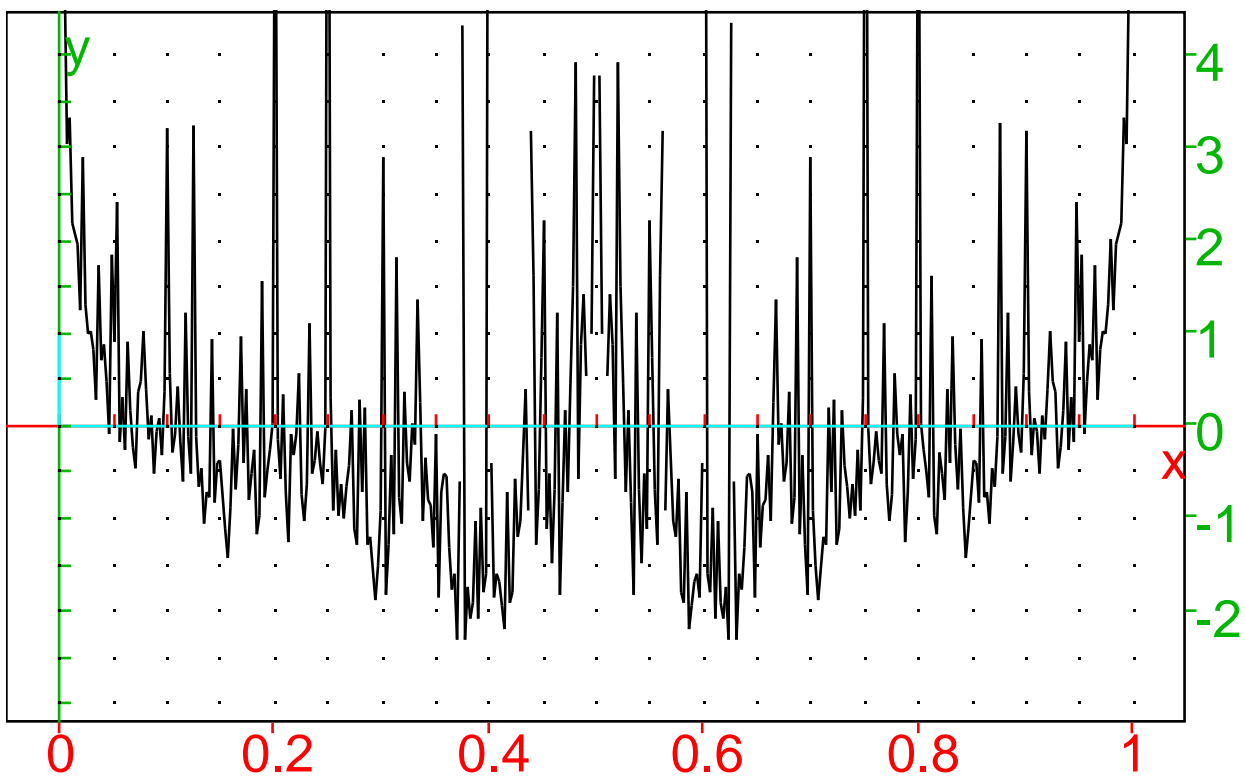

FIGURE $6 . \Phi_{\frac{1}{10}, 50000}$

features: we "see" that $\mathscr{G}_{s, N}(\alpha)$ converges for less values of $\alpha$ when $s$ is getting closer to 0 ) and also that the absolute maximun of $\mathscr{G}_{s}$ seems to be achieved at $\frac{\sqrt{5}-1}{2}$ and $\frac{3-\sqrt{5}}{2}$. 


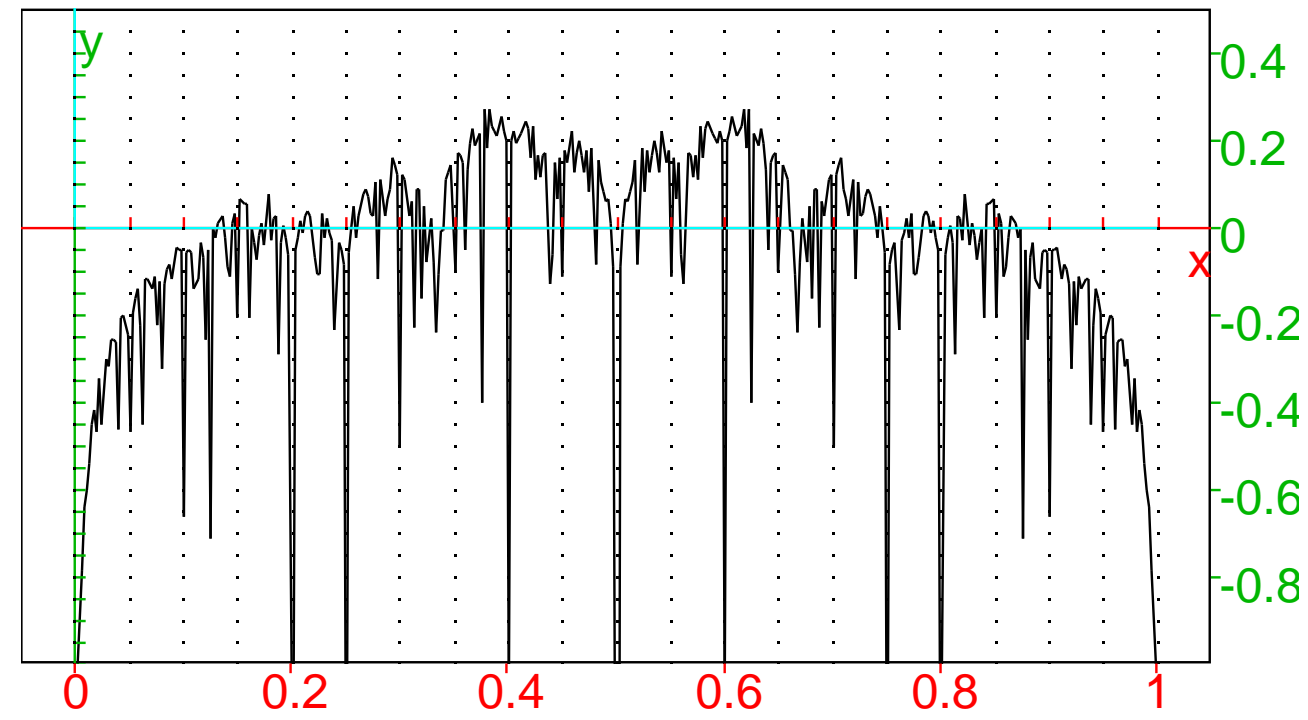

FIGURE 7. $\mathscr{G}_{\frac{1}{2}, 200}$

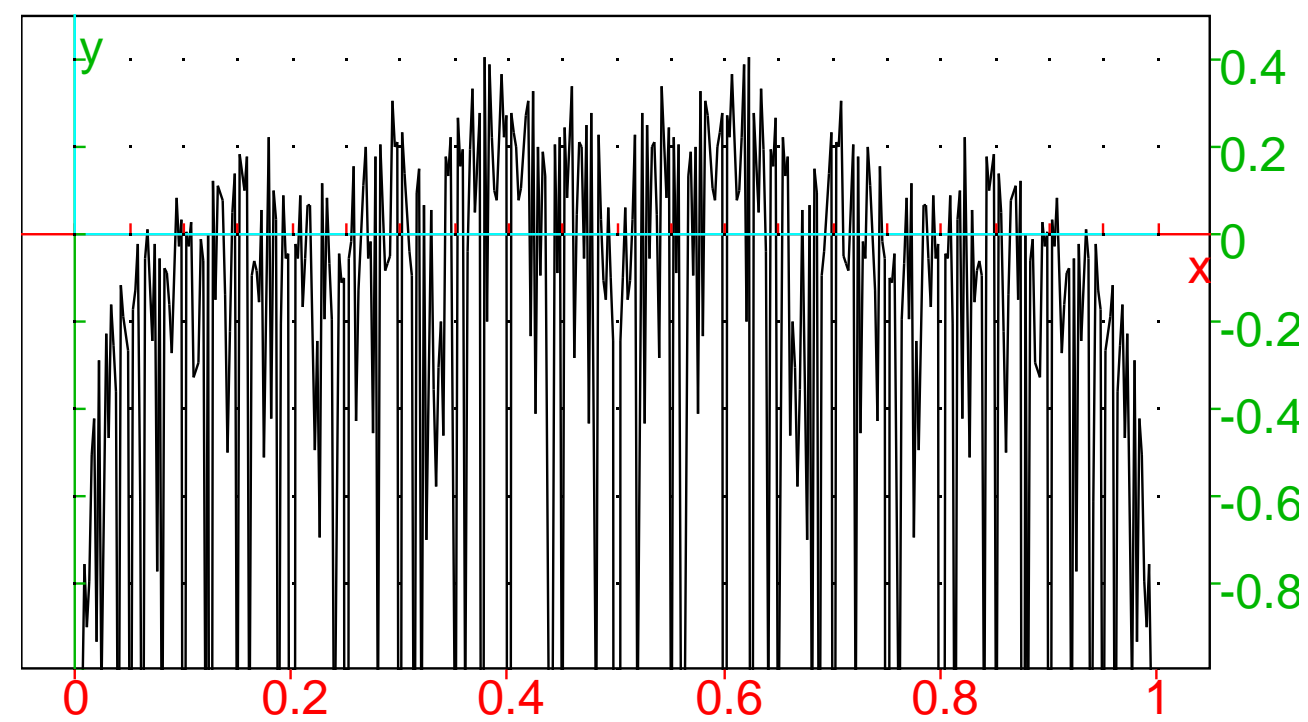

FiguRE 8. $\mathscr{G}_{\frac{1}{10}, 200}$

\section{OPEN PROBLEMS}

We collect here some questions that we believe are interesting and worth investigating. The answer might depend on the value of $s \in(0,1]$.

1) To find the exact domains of definition of $\Phi_{s}$ and $\mathscr{G}_{s}$.

2) To prove or disprove that $\Phi_{s}$, resp. $\mathscr{G}_{s}$, is bounded below, resp. above, on its domain of definition. To prove or disprove that $\Phi_{s}$, resp. $\mathscr{G}_{s}$, is not bounded above, resp. below. 
3) To prove or disprove that $\Phi_{s}$, resp. $\mathscr{G}_{s}$, takes its minimum, resp. maximum, at $\frac{\sqrt{5}-1}{2}$ and $\frac{3-\sqrt{5}}{2}$.

4) To improve the bounds in Propositions 2 or to device a different and more efficient method to compute approximate values of $\Phi_{s}$ and $\mathscr{G}_{s}$.

5) To find expressions of $\liminf _{n} F_{n}(\alpha)$ and $\limsup _{n} F_{n}(\alpha)$ in terms of some known diophantine characteristics of $\alpha$. The techniques of [3] and [19] might be useful here.

6) For some given $s<1$, consider $\alpha, \beta \in \mathscr{A}_{s}$ such that $\Phi_{s}(\alpha) \leq \Phi_{s}(\beta)$. Do we have $\Phi_{t}(\alpha) \leq \Phi_{t}(\beta)$ for any $t \in(s, 1]$ ? Same question with $\mathscr{G}_{s}$ instead of $\Phi_{s}$.

7) Do the graphs of $\Phi_{s}$ and $\mathscr{G}_{s}$ have any kind of fractal/auto-similarity structure, even in an approximate sense? For this problem, does the similarity of the Fourier coefficients of $\Phi_{s}$ with those of Eisenstein series can be used in some way? We recall for example that Itatsu [11] used theta series to study the differentiability of Riemann's function $\sum_{n=1}^{\infty} \frac{\sin \left(n^{2} \alpha\right)}{n^{2}}$. See also [8].

8) Since $\cos (\pi n(n+1) \alpha)=\cos (\pi n \alpha) \cos \left(\pi n^{2} \alpha\right)-\sin (\pi n \alpha) \sin \left(\pi n^{2} \alpha\right)$, we have

$$
\Phi_{s}(\alpha)=\frac{1}{2} \sum_{n=1}^{\infty} \frac{\cot (\pi n \alpha) \sin \left(2 \pi n^{2} \alpha\right)}{n^{s+1}}-\sum_{n=1}^{\infty} \frac{\sin \left(\pi n^{2} \alpha\right)^{2}}{n^{s+1}} .
$$

The second series on the right hand side converges for any $\alpha$ and any $s>0$ and is reminiscent of Riemann's function. The first series seems to attain its minimum at $\frac{\sqrt{5}-1}{2}$ when $s \in(0,1]$. Is this true?

In connection with the last two questions, we observe that the implied series have a ressemblance with the one on the left hand side of the identity

$$
\sum_{\substack{m \in \mathbb{Z} \\ m \text { odd }}}(-1)^{(m-1) / 2} q^{m^{2} / 4} \cot \left(\frac{m \pi \alpha}{2}-\pi x\right)=-i \frac{\theta_{3}(\alpha) \theta_{4}(\alpha) \theta_{2}(\alpha)}{\theta_{4}(x, \alpha)}
$$

where $q=e^{i \pi \alpha}$ and

$$
\begin{gathered}
\theta_{3}(x, \alpha)=\sum_{m \in \mathbb{Z}} q^{m^{2}} e^{2 i \pi m x}, \quad \theta_{4}(x, \alpha)=\sum_{\substack{m \in \mathbb{Z} \\
\theta_{2}(x, \alpha)}}(-1)^{m} q^{m^{2}} e^{2 i \pi m x} \\
\theta_{\substack{m \\
\text { odd }}} q^{m^{2} / 4} e^{i \pi m x}, \quad \theta_{1}(x, \alpha)=\sum_{\substack{m \in \mathbb{Z} \\
m \text { odd }}}(-1)^{(m-1) / 2} q^{m^{2} / 4} e^{i \pi m x}
\end{gathered}
$$

are the usual Jacobi theta series. The modular properties of the function in (9.1) and of many other related functions are studied in [16].

Finally, let us mention that the related series (amongst many others)

$$
\sum_{n=1}^{\infty} \frac{\sin (2 \pi n\|n \alpha\|)}{n^{2} \sin (\pi\|n \alpha\|)}, \quad \sum_{n=1}^{\infty} \frac{\sin (2 \pi n\|n \alpha\|)}{n^{2}\|n \alpha\|}, \quad \sum_{n=1}^{\infty} \frac{\left\|n^{2} \alpha\right\| \sin ^{2}\left(\pi n^{2} \alpha\right)}{n^{2}\|n \alpha\|}
$$


all seem to be extremal at $\alpha=\frac{\sqrt{5}-1}{2}$. On the other hand, the series $\sum_{n=1}^{\infty} \frac{\left\|n^{2} \alpha\right\|}{n^{2}\|n \alpha\|}$ seems to be minimal at $\sqrt{5}-2$; it is studied in details [20], along with the series $\sum_{n=1}^{\infty}(-1)^{n} \frac{\left\|n^{2} \alpha\right\|}{n^{2}|| n \alpha \|}$ which seems to be minimal at $\sqrt{2} / 2$.

\section{REFERENCES}

[1] A. Baker, Rational approximations to $\sqrt[3]{2}$ and other algebraic numbers, Quart. J. Math. Oxford Ser. (2) 15 (1964), 375-383.

[2] C. Baxa, Comparing the distribution of (no)-sequences, Acta Arith. 94 (2000), 345-363.

[3] T. C. Brown, P. J-S. Shiue, Sums of fractional parts of integer multiples of an irrational, J. Number Theory 50 (1995), no. 2, 181-192.

[4] P. Bundschuh, Irrationalitätsmaße für $e^{a}, a \neq 0$ rational oder Liouville-Zahl, Math. Ann. 192 (1971), 229-242.

[5] L. Carleson, On convergence and growth of partial sums of Fourier series, Acta Math. 116 (1966), $135-157$.

[6] R. M. Corless, G. H. Gonnet, D. E. G. Hare, D. J. Jeffrey, D. E. Knuth, On the Lambert $W$ function, Adv. Computational Maths. 5 (1996), 329-359.

[7] T. W. Cusick, M. E. Flahive, The Markoff and Lagrange spectra, Mathematical Surveys and Monographs, 30. American Mathematical Society, Providence, RI, 1989. x+97 pp.

[8] J. J. Duistermaat, Selfsimilarity of "Riemann's Nondifferentiable Function", Nieuw Arch. Wisk. (4) 9 (1991), no. 3, 303-337.

[9] M. Z. Garev, On a multiple trigonometric series, Acta Arith. 102 (2002), no. 2, 183-187.

[10] GP-Pari, freely available at http://pari.math.u-bordeaux.fr

[11] S. Itatsu, Differentiability of Riemann's function, Proc. Japan Acad. Ser. A Math. Sci. 57 (1981), no. 10, 492-495.

[12] H. Jager, J. de Jonge, The circular dispersion spectrum, J. Number Theory 49 (1994), no. 3, 360-384.

[13] A. Ya. Khintchine, Continued fractions, Dover Publications, 1997.

[14] A. H. Kruse, Estimates of $\sum_{k=1}^{N} k^{-s}\langle k x\rangle^{-t}$, Acta Arith. 12 (1966/1967), 229-261.

[15] M. Mignotte, Approximations rationnelles de $\pi$ et quelques autres nombres, Journées Arithmétiques de Grenoble 1973, Bull. Soc. Math. France, Mem. 37 (1974), 121-132.

[16] L. J. Mordell, The definite integral $\int_{-\infty}^{\infty} \frac{e^{a x^{2}+b x}}{e^{c x}+d} d x$ and the analytic theory of numbers, Acta Math. 61 (1933), 323-360.

[17] N. I. Fel'dman, Yu. V. Nesterenko, Transcendental numbers, translated in english from the Russian by Neal Koblitz. Encyclopaedia of Mathematical Sciences 44. Berlin: Springer. 345 p.

[18] H. Niederreiter, On a measure of denseness for sequences Topics in classical number theory, Vol. I, II (Budapest, 1981), 1163-1208, Colloq. Math. Soc. Jnos Bolyai, 34, North-Holland, Amsterdam, 1984.

[19] C. G. Pinner, On sums of fractional parts $\{n a+b\}$, J. Number Theory 65 (1997), 48-73.

[20] T. Rivoal, Extremality properties of some diophantine series, 24 pages (2009), to appear in Experiment. Math.

[21] G. Tenenbaum, Introduction à la théorie analytique et probabiliste des nombres, Deuxième édition. Cours Spécialisés, 1. Société Mathématique de France, Paris, 1995. xv+457 pp.

[22] XCAS, freely available at http://www-fourier.ujf-grenoble.fr/ parisse/giac_fr.html.

T. Rivoal, Institut Fourier, CNRS UMR 5582, Université Grenoble 1, 100 Rue des Maths, BP 74, 38402 Saint-Martin D'Hères CEDEX, France.

WWW: http://wwW-fourier.ujf-grenoble.fr/ ${ }^{\sim}$ rivoal. 